\title{
Paper Location Estimation of Nodes in Underwater Acoustic Sensor Networks
}

\author{
B. S. Halakarnimath and A. V. Sutagundar \\ ${ }^{1}$ Research Scholar of VTU, Department of Computer Science and Engineering, S.G. Balekundri Institute of Technology, \\ Belagavi, Karnataka, India \\ ${ }^{2}$ Department of Electronics and Communication, Basaveshwar Engineering College, Bagalkot, Karnataka, India
}

https://doi.org/10.26636/jtit.2021.145720

\begin{abstract}
The paper presents a location estimation scheme for underwater acoustic sensor networks. During the first phase, the sink node begins the trapezoid formation process by activating the trapezoid formation agent. It stores relevant information in the sink's knowledge base and in the node's knowledge base, and also develops the search data structure required for locating the node. During the second phase, the position of the node is determined by utilizing the search data structure. Identification of the location of all nodes by traveling across the trajectory may be performed as well, as an alternative approach. When identifying the location of one node, the estimation is performed based on the search data structure. When determining the position of all nodes, the sink node agent travels along the defined trajectory and transmits beacon messages which contain the real-time location at specific points. The anchor node agent measures the signal strength and localizes itself and begins estimating the locations of other nodes within the trapezoids, using location estimation techniques. Various performance parameters are used to validate the proposed scheme.
\end{abstract}

Keywords—location estimation, trapezoid, UASN.

\section{Introduction}

Location estimation for large scale mobile underwater acoustic sensor networks (UASNs) is intriguing because of harsh aqueous environments. Even though acoustic methods are suitable for underwater communication, such features as moderate bandwidth and considerable failure rate impose specific constraints on location estimation schemes [1]. Propagation delays, movement-caused Doppler shifts, amplitude and phase fluctuations, and multipath obstruction are all factors that need to be taken into consideration in location determination procedures. Some of the localization-related issues are presented below [2]:

- need for a proper sound-speed variation model used for location estimation,

- immersed sensor nodes need precise time synchronization,

- efficient node mobility pattern for dynamic underwater conditions,
- impacts related to medium access control (MAC), including contention fixing, transmission overhead, localization accuracy and latency,

- implications of location estimation protocols for location-based routing and clustering techniques.

In this paper, a computational geometrical-based localization technique is presented. The proposed location estimation scheme works in the following manner.

During the first stage, the sink node begins the trapezoid formation process on the sea surface by activating the trapezoid formation agent (TFA) and by deploying an autonomous underwater vehicle (AUV) to reach a particular depth below the surface of the sea. The AUV travels across the linear trajectory of a fixed length, at a specific depth, and the TFA creates trapezoids in the upper and lower portions of the path. The TFA stores the relevant information in the sink knowledge base (SKB) and in the node knowledge base (NKB). In the meantime, the TFA develops also the SDS in order to locate the node in the easiest manner possible.

During the second phase, position of the node is determined by relying on two methods. The first method determines the node's location by utilizing the SDS, and the other consists in finding the location of all the nodes by traveling across the trajectory. In any case, the sink triggers the localization agent (LA) and deploys the AUV to a specific depth under the surface of the ocean.

In the case of finding the location of one node, the LA moves directly to the trapezoid, which is given per the SDS, and performs the localization process.

In the case of determining the position of all nodes, the AUV traverses along the continuing trajectory and transmits beacon messages which contain real-time locations at specific points. The anchor agent (AA) at the anchor node receives these beacon messages, measures the signal strength and localizes itself based on the position of the broadcast point and the received signal strength. The LA begins the location estimating activity with the associated trapezoids of the anchor nodes, relying on location estimation techniques.

All agents keep updating the information to the respective knowledge bases whenever the data is modified. 


\section{Problem Statement}

In a fixed area water network $O, n$ uw-sensor nodes are randomly placed. $m$ is the number of reference nodes with known locations and $N$ is the total number of nodes existing in the network, where $N=n+m$. For a 2D localization problem $N=\left[N_{x}, N_{y}\right]$, where $N_{x}=\left\{x_{1}, x_{2}, \ldots\right.$, $, x_{n}, \quad N_{y}=\left\{y_{1}, y_{2}, \ldots, y_{n}\right\}$ and where the reference nodes' coordinates are $N_{x}=\left\{x_{n+1}, x_{n+2}, \ldots, x_{n+m}\right\}, N_{y}=$ $\left\{y_{n+1}, y_{n+2}, \ldots, y_{n+m}\right\}$. The location of each uw-sensor node $i$ is appended with a third coordinate $Z_{i}$, being the depth of each uw-sensor node. The $2 \mathrm{D}$ problem is extended to $3 \mathrm{D}$ by appending the third coordinate to each uw-sensor node location. The $n_{x, y}$ measurement could be a physical reading indicating the relative position. Process the monitoring area of ocean $O$, and divide the network into trapezoids of variable sizes, find the trapezoid of $O$ containing the uw-sensor node $u w_{i}$, and then estimate the location of unlocalized $u w_{i}$ within that trapezoid. The aim is to design and simulate the above task by creating trapezoids to facilitate efficient localization by considering the dynamic characteristics of the ocean.

In the proposed location-estimation scheme, our research additions are as follows:

- Setting up a network to perform the localization process.

- Applying a computational geometry-based trapezoidal map forming numerous trapezoids of distinct shapes.

- Developing the AUV's path of travel and iteratively submitting real-time position information.

- Creating appropriate node agencies for the proposed MASD scheme.

- Developing methods for trapezoid formation, singlenode location estimation, and for localization of all nodes.

- Simulating the proposed location estimation scheme.

- Evaluating the proposed MASD scheme based on different performance parameters.

\section{Related Work}

Range-free and range-based techniques are the two primary classifications of location estimation techniques [3]. The range-based schemes, such as time of arrival (ToA), time difference of arrival (TDoA), angle of arrival (AoA), and received signal strength indicator (RSSI) provide a relatively precise location compared with range-free schemes. The speed of underwater sound propagation encourages the employment of range-based schemes for underwater environments. The speed of sound depends on salinity, density, and temperature. It changes continuously in undersea conditions. Hence, an accurate time synchroniza- tion model is also required. Assume that the sound speed change remunerated by applying signal processing methods. Range-based systems, such as TDoA and ToA, achieve relatively high accuracy levels but require more real-time synchronization within uw-nodes, which increases the cost of UASNs due to the additional hardware needed. Rangefree location finding algorithms are accessible if high localization accuracy levels are not essential. In rangefree schemes, neighboring ranges or angle learning is challenging to measure due to hardware limitations. Once the range between uw-nodes has been estimated, rangefree techniques rely on trilateration to determine precise locations.

In [4], energy models of acoustic waves are applied to determine the location of acoustic sensors in physical networks. For the calculation of a specific target position, efficiency and impact analyses are performed by applying the Cramer-Rao bound (CRB). The ML approach provides exceptionally reliable results and an enhanced level of capacity for location estimation based on multiple sources. This approach is scalable and may cover more targets within a predefined sensor area. The scheme requires some improvement in terms of parameter sensitivity analysis and sequential Bayesian estimation. The authors proposed a nondistributed range-free method in [5] that presents a rough location calculation of a sensor within a particular area, instead of its exact position. A sensor node overhears beacons from various anchor nodes and records their power levels independently, measuring also the mode used by the recorded power signals of each anchor node. Once collected, the information is transmitted to the onshore sink to identify the area in which the anchor node is present. This approach is manageable, synchronization free and cooperative. It is also resistant to changes in the speed of sound.

In [6], an event-driven iterative distributed location estimation method is proposed that produces excellent throughput, still retaining a moderate percentage of beacon nodes. The mobility model is a shortfall of this work. Localization becomes more comfortable in undersea conditions if anchor/reference nodes are available within the network [7]. The link is adopted to succeed in the failures linked to the balance of the line of sight (LOS). Underwater signal reflection-enabled acoustic-based localization (UREAL) is suitable for networks operating in shallow water environments. It offers the use of multi-modal directional undersea piezoelectric transducers that are relied upon to create either directional or omnidirectional beacons. To distinguish between LOS/NLOS, RSSI is applied. To calculate the location, AoA is applied. This scheme is independent of the LOS link in performing the location estimation process. The finite difference time domain (FDTD) approach is applied to estimate the reflection points for non-line of sight (NLOS) positioning.

In [8], collision-free and collision-tolerant packet scheduling techniques are proposed for location estimation in single-hop underwater networks. Experimental analysis 
proves that sophisticated collision design takes less time than its collision-free counterpart, when the average probability of packet loss is close to one. Authors in [9] describe different deployment approaches and their impacts on localization-related performance. They consider the tetrahedron deployment scheme for a 3D environment that performs better than cube deployment and random deployment schemes in terms of providing better localization ratios, minimizing localization errors, and maintaining better network connectivity. They emphasize that their future work should focus on designing a realistic model that considers the various conditions experienced in underwater environments, including mobility issues, sound speed variations and propagation losses. Harsh underwater environments require cooperation between the nodes for broader coverage and better accuracy in identifying locations of the nodes.

For NLOS localization, the least square cooperative localization method is considered in [10]. The authors analyze consistency and efficiency of least square cooperative localization. The Fisher information matrix (FIM) is derived for an NLOS bios model and proves that Gaussian bias produces the worst-case scenario, as well as that lower partial ordering leads to the largest FIM.

RSS-based localization for UASNs is proposed in [11]. The system model considers various attenuation parameters, spreading losses and issues related to the environment, in order to account for acoustic propagation losses. The author examines semi-definite programming with frequencydependent RSS and RSS based to yield better localization performance.

The method known as localization technique for underwater sensor networks (LOTUS) [3] estimates the nodes' approximate location based on two reference/anchor nodes only, by using fewer iterations and with local interactions. Experimental results justified a reduction in overhead, improved localization coverage and minimum localization error compared with large-scale localization. A collaborative or n-hop multilateration primitive for higher accuracy in two computation models, i.e. the centralized and the distributed model, is presented in [12]. An atomic/collaborative multilateration used iteratively to compute the locations of unlocalized uw-sensors is adopted in [13]. The method presented in [14] employs two-phase localization, i.e. anchor node location estimation and other normal node localization based on mobility prediction. Parameters of mobility patterns are predicted by using the covariance algorithm. Prediction errors are minimized by employing the covariance algorithm.

In [15], the author presents a localization method using the mobile beacon (LoMoB) range-free algorithm. Sensor nodes receive bacon messages which contain location information, and are localized without communicating with other nodes. The beacon points are projected on the sensor nodes' three-dimensional horizontal plane. Once the projection is made, the 3D localization problem is converted into a 2D localization problem. The sensor node's location is estimated based on the potential locations, by using a weighted mean of those potential locations. The author compares his scheme with LDB and shows a significant improvement in localization accuracy. The RSSI-assisted mobile anchor node location determination scheme is proposed in [16], aiming to reduce the location lead time and to enhance the level of location accuracy. Using the mobile anchor node's trajectory, projection of the sensor node is estimated by using the interpolation method supporting vector regression, which improves location accuracy. A curve matching method is designed to reduce the localization lead time and to obtain the perpendicular distances, along the mobile anchor node's linear trajectory, from each sensor node. The benefit of this scheme is that it requires a one-time trajectory for the mobile anchor node to locate other sensor nodes. To improve location accuracy even further, the error within the actual perpendicular distance and the estimated perpendicular distance in the curve matching method could be reduced.

The multi-anchor nodes' collaborative localization (MANCL) [17] method classifies the entire localization process into four sub-processes: ordinary node location estimation process, iterative location estimating method, enhanced 3D Euclidean distance calculation method, and 3D DV-Hop distance calculation method based on two-hop anchor nodes. The enhanced 3D Euclidean distance calculation process applies the transmission mechanism or the voting mechanism to estimate the transient locations of regular sensor nodes. During the 3D DV-Hop distance calculation process, the ordinary node's coordinates are calculated based on the average two-hop anchor node distance.

The double rate localization (DRL) method is described in [18], relying on one anchor for performing localization in multi-hop underwater acoustic networks (UANs). In this scheme, the localization process is divided into high-rate and low-rate transmission modes by selecting an appropriate bit duration to ensure the transmission rate and to improve the accuracy of range measurements in multi-hop networks. Optimized selection of reference nodes improves the accuracy of localization performed with the help of one anchor.

Most localization algorithms are synchronized with the time frame, and it is not very easy to achieve accurate times. In [19], the author proposes a localization scheme without any time synchronization. The existing dive and rise (DNR) scheme is enhanced for the purpose of the localization process by excluding time synchronization. A specific anchor node dives vertically and broadcasts beacon messages at regular intervals to localize the sensor nodes. After a specific time, the anchor node rises vertically, broadcasting the beacon messages. This entire process allows to identify the distance between the anchor node and the nodes within the transmission area, thus estimating the nodes' position. This scheme assumes that the nodes are motionless, which is impractical.

In [20], the authors develop a multi-period particle swarm optimization (MP-PSO) algorithm that analyzes water mo- 
bility patterns the seashore, for the purpose of the localization process. The beacon nodes are located, and their velocities are estimated using the range-based PSO algorithm. Initially, the spatial mobility correlation of the underwater objects (the nodes) is applied to estimate the velocity of the undiscovered nodes and, their locations are predicted thereafter. This algorithm offers more reliable localization coverage and enhanced localization accuracy. Computation complexity and energy consumption of this algorithm are relatively high.

Papers [2], [4], [8], [9] focus primarily on traditional methods used for determining node locations, neglecting undersea conditions. Their authors present numerous techniques that provide more insight into such issues as time synchronization, network lifetime enhancement, as well as mobility in localization processes, with empirical results shown, too.

Most of the existing algorithms focus on estimating the nodes' location by relying on autonomous underwater vehicles, anchor nodes using geometrical structures, but neglect the mobility of the nodes and their energy efficiency. The survey shows also that computational geometry is to relied upon while estimating node locations. There was a lack of focus on the adoption of computational geometrical fundamental approaches in localization schemes. This factor motivates us to design and simulate a geometrically-based mechanism for forming trapezoids and finding the nodes' location, with the dynamic sea conditions taken into consideration. In article [21], a review has been conducted, revealing the existing difficulties encountered in marine environments. In the review, the UWSN is introduced initially. Then, basic information concerning underwater conditions and the localization technique are discussed. After that, the article focuses on the UWSN architecture and on the technologies used for localization purposes. Several centralized and distributed localization techniques are presented in the paper. The estimation- and prediction-based localization algorithms are presented as well. The localization algorithms are grouped based on range-free and range-based schemes. Finally, the article focuses on the difficulties impacting underwater acoustic communications and underwater localization.

\section{Proposed Work}

In this section, the proposed localization scheme using computational geometry is presented. Though this work focuses mainly on localization in 3D network architectures, some issues, such as cluster creation, energy usage and topology control are intrinsic. These issues play a significant role in creating an efficient location estimation method. This section described the network architecture, the trilateration method, the agent technology, and the localization scheme under consideration.

\subsection{Network Environment}

In two-dimensional UASN, sensor nodes are grounded at the bottom of the sea. In the $3 \mathrm{D}$ variant, underwater sensor nodes are deployed at various depths in order to cover the desired area. The 3D UASN that this work is concerned with consists of AUVs, ordinary uw-sensor nodes, and anchor nodes. Each node may operate in different modes. In its active state, the node operates with full functionality. In the semi-active state, the sensor node is capable of sensing and receiving signals. In the inactive state, the node is in not in the operating state, i.e. its residual energy level is below the threshold value. It is assumed that uw-nodes are allowed to transmit to/from anchor/reference nodes only. The sink node controls the operation of AUV and obtains information that tasks have been performed. Some anchor/reference that are more capable than uw-sensors are randomly deployed at various sea levels and are motionless. The nodes are deployed uniformly and randomly throughout the targeted section of the sea. Each node is capable of communicating, may be anchored, and is movable. One sink node is installed onshore to control all activities performed.

The range of capabilities of AUVs is higher than in the case of uw-sensor and anchor nodes. The network model consists of a few AUVs to avoid the extra costs. Uw-sensors are deployed randomly by dropping them in the water. Uwsensor and anchor nodes collectively make up an ad-hoc network operating on plane $O$. When the AUV is deployed at a certain depth and travels, and it may calculate its coordinates. In this work, the AUV helps the nodes estimate their location. Some reference/anchor nodes are employed on the seaplane to assist in the localization process. The network environment is shown in Fig. 1. The monitored are of ocean $O$ is a planar subdivision with the size of $O_{l} X O_{b} X O_{d}$. The water is divided into many vertical levels based on the transmission range of AUV as:

$$
O_{V L}=\frac{O_{d}}{A U V_{C R}} .
$$

At each vertical layer, AUVs are deployed at a fixed depth. AUVs are proficient in acquiring their locations directly

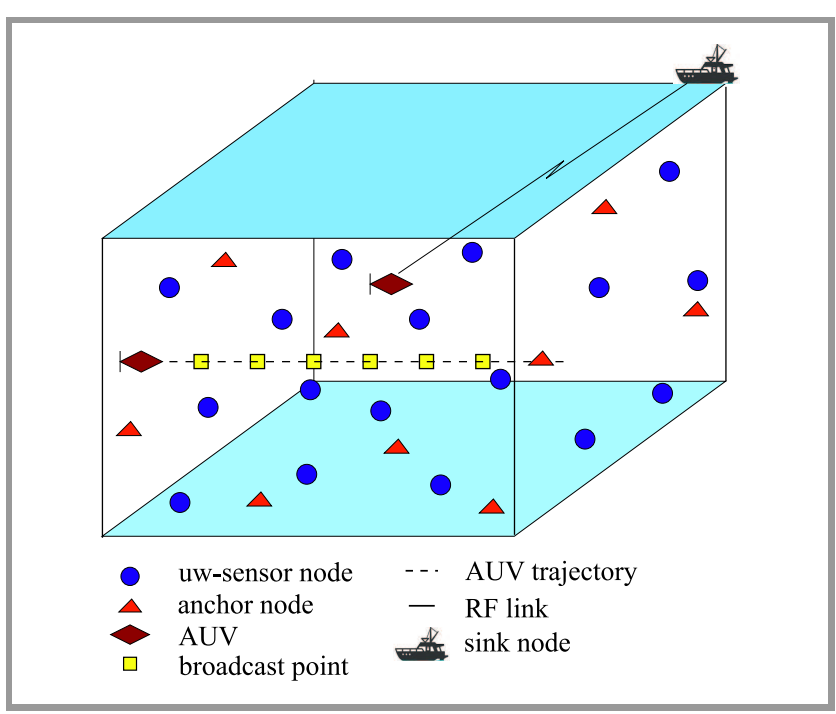

Fig. 1. The UASN network architecture. 
Table 1

Abbreviations used

\begin{tabular}{|l|c|}
\hline \multicolumn{1}{|c|}{ Definition } & Notation \\
\hline \hline Absorption model & $\alpha(\mathrm{f})$ \\
Autonomous underwater vehicle & AUV \\
Anchor ID & $A_{I D}$ \\
Trapezoid ID of anchor & $A_{N T}$ \\
Neighbors of anchor's node & $A_{N N T}$ \\
Residual energy & $A_{E R}$ \\
Position of anchor & $A_{(X, Y, Z)}$ \\
AUV communication range & $A U V_{c r}$ \\
Directive index & $\mathrm{DI}$ \\
Detected threshold & $\mathrm{DT}$ \\
Node energy threshold & $E_{t h}$ \\
Geometrical spreading factor of propagation & $\mathrm{k}$ \\
Length of the AUV's linear trajectory & $\mathrm{L}$ \\
Neighbor count & $N_{C}$ \\
Node depth & $N_{D}$ \\
Node ID & $N_{i d}$ \\
Node's trapezoid ID & $N_{t i d}$ \\
Node position & $N_{(x, y, z)}$ \\
Noise level & $\mathrm{NL}$ \\
Propagation loss & $\mathrm{PL}$ \\
Source level & $\mathrm{SL}$ \\
\hline
\end{tabular}

from GPS. Assuming that the outline of each trapezoid $T_{i}$ or $T_{j}(i, j \leq n)$ is disjoint without gaps and overlaps, i.e. the trapezoid is located between $T_{i, j} \subseteq O$ and $T_{i} \cap T_{j}=\emptyset$, where $i, j \leq n$. Since the AUV may transmit in all directions, sensor nodes may be located in the upper and lower portions of its linear trajectory. Anchor/reference nodes acquire their positions whenever they are within the AUV's transmission range, by using the RSSI technique. Let us assume that the deployment of anchor nodes forms a planar graph $G(V, E)$, where reference/anchor nodes are the vertices, and communication edges between these nodes are the segments/edges. Table 1 presents the abbreviations used in this work.

\subsection{Trilateration Method}

Trilateration is a process of finding position by measuring the distance using signal strengths from different sources. In a trilateration scheme, the location of a uw-sensor $u_{i}$ may be found if at least three localized nodes (signal sources) or reference nodes appear in the proximity of $u_{i}$. As shown in Fig. 2a, $L_{1}, L_{2}$, and $L_{3}$ are three localized uw-sensors with known locations, present in the proximity of an unlocalized uw-sensor node $u$. It is possible to determine the distance of an object if it remains within the sensing range centered around $L_{1}$ or $L_{2}$ or $L_{3}$. Let $u$ be the un-localized node with location $\left(x_{u}, y_{u}\right)$ and let be the known locations of $\left(x_{L 1}, y_{L 1}\right),\left(x_{L 2}, y_{L 2}\right)$, and $\left(x_{L 3}, y_{L 3}\right), L_{1}, L_{2}$, and $L_{3}$, respec- (a)

(b)

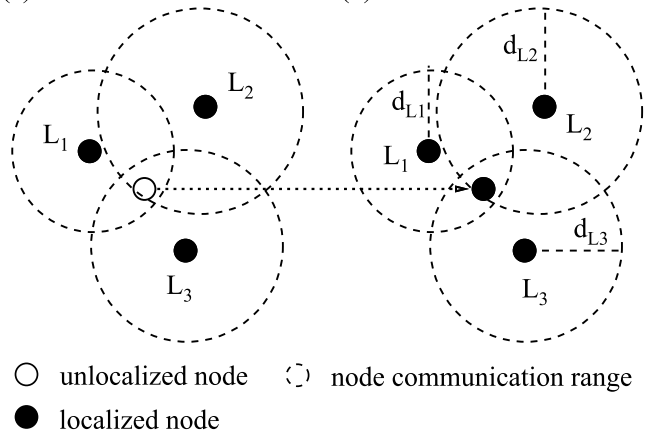

Fig. 2. Network: (a) before trilateration and (b) after trilateration.

tively. Let $d_{1}, d_{2}$, and $d_{3}$ be distances from the unknown object, respectively. The distance equations are:

$$
\begin{aligned}
& \left(x_{u}-x_{L 1}\right)^{2}+\left(y_{u}-y_{L 1}\right)^{2}=d_{1}^{2}, \\
& \left(x_{u}-x_{L 2}\right)^{2}+\left(y_{u}-y_{L 2}\right)^{2}=d_{2}^{2}, \\
& \left(x_{u}-x_{L 3}\right)^{2}+\left(y_{u}-y_{L 3}\right)^{2}=d_{3}^{2} .
\end{aligned}
$$

By expanding the squares in each equation:

$$
\begin{aligned}
& x_{u}^{2}-2 x_{u} x_{L 1}+x_{L 1}^{2}+y_{u}^{2}-2 y_{u} y_{L 1}+y_{L 1}^{2}=d_{1}^{2}, \\
& x_{u}^{2}-2 x_{u} x_{L 2}+x_{L 2}^{2}+y_{u}^{2}-2 y_{u} y_{L 2}+y_{L 2}^{2}=d_{2}^{2}, \\
& x_{u}^{2}-2 x_{u} x_{L 3}+x_{L 3}^{2}+y_{u}^{2}-2 y_{u} y_{L 3}+y_{L 3}^{2}=d_{3}^{2} .
\end{aligned}
$$

From Eqs. (5)-(6):

$$
\begin{aligned}
\left(-2 x_{L 1}+2 x_{L 2}\right) x_{u}+ & \left(-2 y_{L 1}+2 y_{L 2}\right) y_{u}= \\
& d_{1}^{2}-d_{2}^{2}-x_{L 1}^{2}+x_{L 2}^{2}-y_{L 1}^{2}+y_{L 2}^{2} .
\end{aligned}
$$

From Eqs. (6)-(7):

$$
\begin{aligned}
\left(-2 x_{L 2}+2 x_{L 3}\right) x_{u}+ & \left(-2 y_{L 2}+2 y_{L 3}\right) y_{u}= \\
& d_{2}^{2}-d_{3}^{2}-x_{L 2}^{2}+x_{L 3}^{2}-y_{L 2}^{2}+y_{L 3}^{2} .
\end{aligned}
$$

Equations (8)-(9) with two unknowns are:

$$
\begin{aligned}
& A x+B y=C, \\
& D x+E y=F,
\end{aligned}
$$

which gives the solution:

$$
\begin{aligned}
& x=\frac{C E-F B}{E A-B D}, \\
& y=\frac{C D-A F}{B D-A E} .
\end{aligned}
$$

The value of the $z$ coordinate is the depth of the node. Figure $2 \mathrm{~b}$ shows the node after the trilateration process. In an ideal case, the intersection of three sensing ranges is the exact location of the object, i.e. node $u$. Once the node computes its location through trilateration, it assumes the 
role of a reference node $\mathrm{R}$ in order to further assist in determining the location of unlocalized nodes. In many cases, distance computations are imprecise, because the sensing range circles may not intersect at a single location. To overcome this error, a maximum likelihood scheme may be adopted to minimize the degree of imprecision in determining the location of a given node.

\subsection{Node Agency}

Node agency is acritical component of each uw-node. It comprises of a static agent NA, and a mobile agent NMA both residing at the node level. It also includes NKB to store node- and trapezoid-related information. Migrant mobile agents TFA and LA perform inter-node communications, assist in location estimation and in the trapezoid formation process. This agency is taking an active part in localization and is also partially involved in the trapezoid formation process. Node agency is shown in Fig. 3.

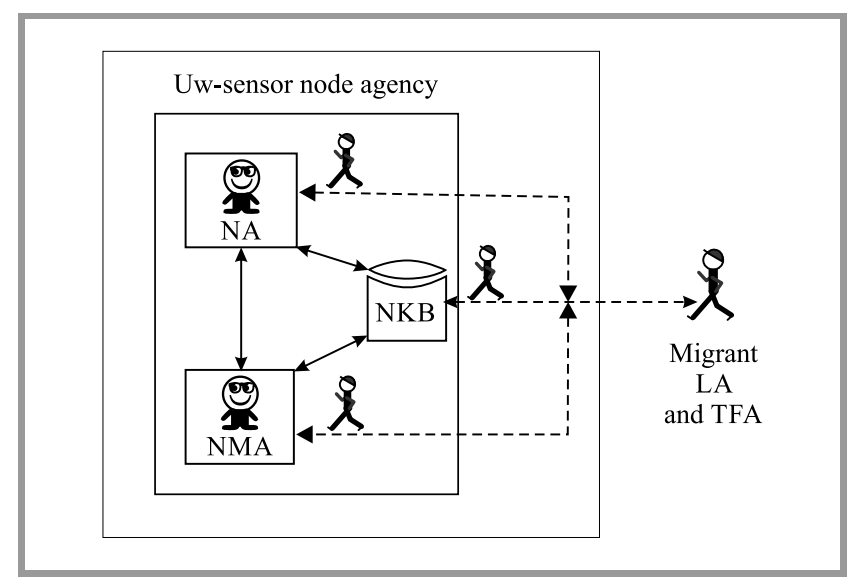

Fig. 3. Node agency.

Node agent (NA) is an autonomous, universal and reactive agent. NA is situated locally at the node level. NA uses NKB to store and retrieve information. NA coordinates with NMA to oversee the activities that are taking place at the uw-sensor node level. This agent communicates with LA to support the location estimation process and interacts with TFA to learn its trapezoid information. NA is responsible for learning which trapezoid it belongs to and for informing node agency when localization is required.

Node manager agent (NMA) is situated in each uw-sensor node of the network. NMA monitors everything that is taking place in and around the node, for taking some decisions and for performing regular activities. Activities performed at the NMA at level include the following:

- changing the node state from semi-active to active and vice-versa,

- to communicating with the outside world in order to synchronize all actions,

- becoming actively involved in the location estimation process with LA,
- effectively managing the node's battery for longer life,

- updating the node knowledge base,

- NMA coordinates with NA, LA, and TFA to complete the tasks.

NMA decides when to estimate its location by verifying the present status of the sensor node.

Node knowledge base (NKB) is a local information storage existing at the level of each node. This knowledge base comprises $N_{I D}, N_{T I D}, N_{E R}, N_{D}, N_{(X, Y, Z)}$, and all information observed. Local and migrant agents communicate with this information storage to store, modify and retrieve the data. The structure of the NKB is given in Table 2.

Table 2

Node knowledge base

\begin{tabular}{|c|c|c|c|c|c|c|}
\hline S1 no. & $N_{I D}$ & $N_{T I D}$ & $N_{E R}$ & $N_{C}$ & $N_{D}$ & $N_{(X, Y, Z)}$ \\
\hline \hline 1 & 24 & 4 & 4.6 & 4 & $203 \mathrm{~m}$ & - \\
\hline 2 & 5 & 6 & 4.76 & 2 & $123 \mathrm{~m}$ & - \\
\hline 3 & 12 & 3 & 4.32 & 3 & $174 \mathrm{~m}$ & - \\
\hline 4 & - & - & - & - & - & - \\
\hline
\end{tabular}

\subsection{Anchor Agency}

Anchor agency comprises a static anchor agent and a mobile anchor management agent (AMA). It includes an anchor knowledge base (AKB) used to store and retrieve information concerning the trapezoids, the node, and the anchor node. Migrant mobile agents - LA and TFA - are used for inter-agent communication in order to perform localization and trapezoid formation processes. This agency plays the key role in creating trapezoids in the target area and participates in the localization process. It stores and updates information in AKB. The anchor agency is shown in Fig. 4.

AUV agent (AA) is an autonomous, universal and reactive agent. It is situated locally at the anchor node level. It uses AKB to store, retrieve and modify information. AA coordinates with AMA to oversee the activities that are taking place at the anchor node level. This agent actively communicates with TFA to create trapezoids. It also communicates with LA and participates in the process of estimating locations within the trapezoids. It stores, in AKB, the trapezoid ID, trapezoid boundary, number of nodes present in the trapezoid, and its association with different trapezoid IDs. AA is responsible for communicating with the sink node in order to identify any variations in the system.

Anchor Manager Agent (AMA) is situated within each anchor node of the network. AMA monitors all activities taking place in and around the anchor node and performs regular tasks. The activities of AMA existing at the anchor node level include the following:

- communicating with the outside world in order to synchronize all actions, 


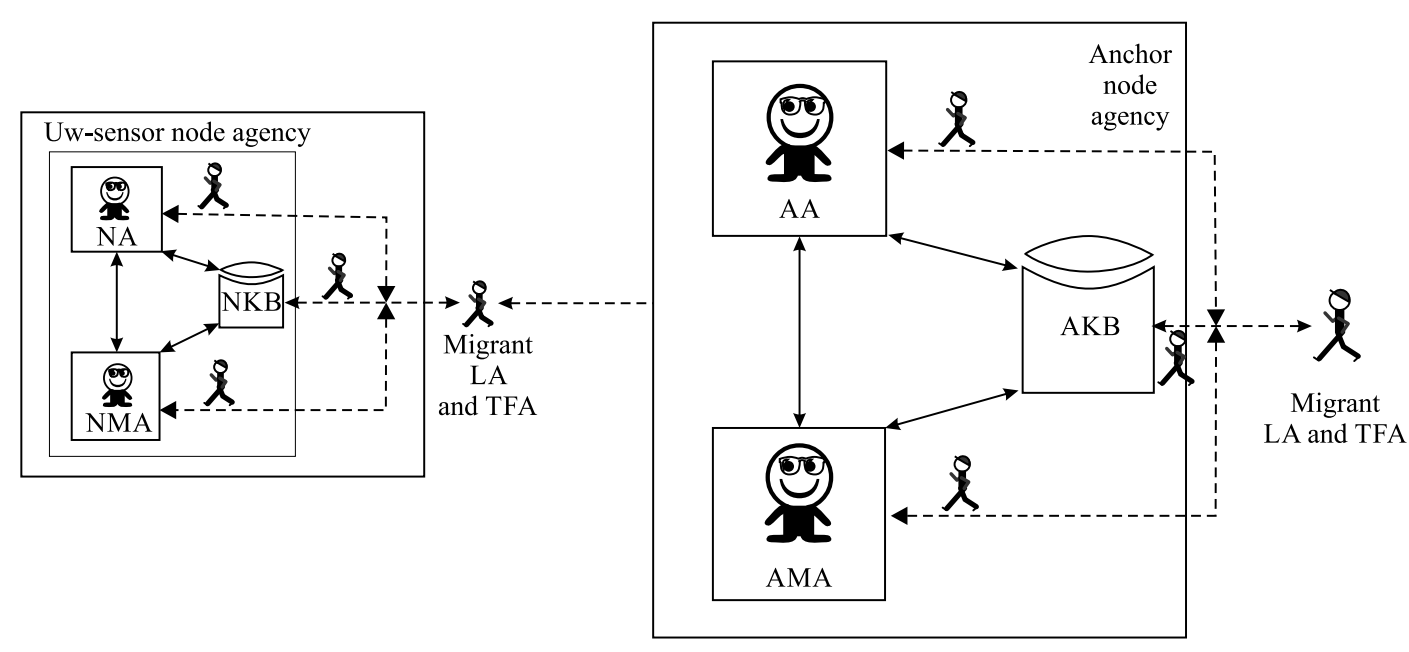

Fig. 4. Anchor node agency.

- becoming actively involved in the location estimation process with LA,

- participating actively in the creation of trapezoids with TFA,

- effectively managing the node's battery for longer life,

- updating the anchor knowledge base,

- AMA coordinates with NA, AA, LA, and TFA to complete the tasks.

AMA informs the sink node about initiating the trapezoid formation process whenever a significant variation takes place concerning the anchor node's location. AMA keeps on updating its position and battery information to the sink node at regular intervals.

Anchor knowledge base (AKB) is a local information storage at each anchor node level. This knowledge base contains $A_{I D}, A_{N T}, A_{N N T}, A_{E R}, A_{(X, Y, Z)}, N_{C}$, and all observed information. Local and migrant agents communicate with this information storage to store, modify and access the data. The structure of NKB is shown in Table 3.

Table 3

Anchor knowledge base

\begin{tabular}{|c|c|c|c|c|c|}
\hline S1 no. & $A_{I D}$ & $A_{N T}$ & $A_{N N T}$ & $A_{E R}$ & $A_{(X, Y, Z)}$ \\
\hline \hline 1 & 3 & 2 & 5 & 4.00 & - \\
\hline 2 & 5 & 3 & 6 & 3.47 & - \\
\hline 3 & 14 & 1 & 2 & 4.72 & - \\
\hline 4 & - & - & - & - & - \\
\hline
\end{tabular}

\subsection{Sink Agency}

Sink agency comprises a static sink management agent (AMA), as well as TFA and LA mobile agents. It uses SKB to save and access information about the entire network.
LA and TFA mobile agents are triggered to perform localization and trapezoid formation processes. Sink agency is the central agency that initiates the trapezoid creation process and the location estimation process. It stores and updates the information in SKB. Anchor agency is shown in Fig. 5.

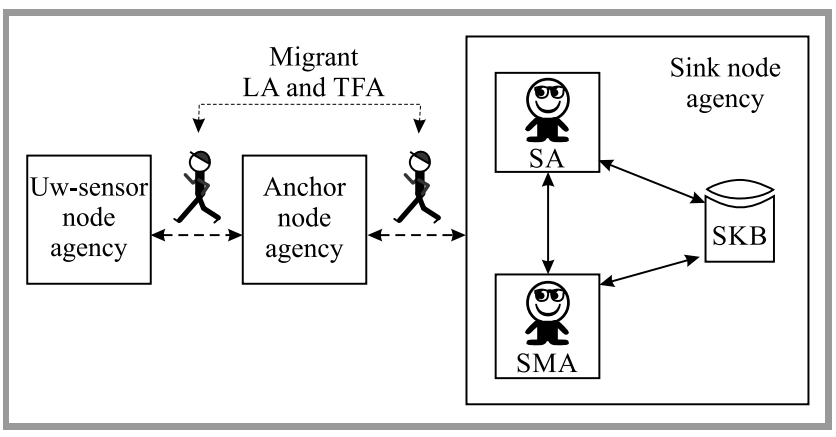

Fig. 5. Sink node agency.

Sink Agent (SA) is an autonomous, universal, adaptive, smart and proactive agent. It is situated locally at the sink node level. It triggers and coordinates LA for localization TFA for trapezoid formation process. It utilizes SKB to save and access information. Sink node communicates with other agencies and agents to accomplish the desired activities, as and when required for the network. The trapezoid formation process is performed for the entire targeted area. On-demand single node or all node localization is performed as and when needed.

Localization Agent (LA) is an autonomous, social, proactive and mobile agent. It interacts with all agents and agencies to collect the required information and to accomplish the localization process. LA is initiated by the sink node to accomplish the on-demand single node or all node localization process and updates the data in all information storages. 
Trapezoid Formation Agent (TFA) is a mobile, selfsufficient, proactive and social agent. The sink node triggers TFA, and its primary objective is to create trapezoids and to construct SDS for a given network. It communicates with all agents and agencies to gather the required information and to accomplish the trapezoid formation process. It creates SDS during the creation of trapezoids. SDS is a tree-based data structure that is better suited for the location of trapezoids.

Sink Manager Agent (SMA) is situated in the network, at the sink node. Its duties include the following:

- communicating with other components of the system and synchronizing the actions,

- intimate sink agent to activate TFA through prelocalization LA, while the localization, and maintain SDS,

- associate in the location estimation process with LA and trapezoid creation process with TFA,

- SMA manages, virtually, the batteries of the uwsensor and the anchor nodes, increasing their life,

- updating the SKB at regular intervals.

Sink Knowledge Base (SKB) is the sink node's information storage, accessible to TFA and LA mobile agents during the trapezoid formation and localization process. This knowledge base contains information on the energy level of the entire network, on AUV's trajectory, on trapezoid information, as well as on the number of active and failed nodes, as shown in Table 4. SA, SMA, LA, and TFA communicate with this information storage to retrieve, modify and store information.

Table 4

Sink knowledge base

\begin{tabular}{|c|c|c|c|c|c|c|}
\hline SN & $A_{I D}$ & $N_{I D}$ & $N_{T I D}$ & $N_{E R}$ & $\mathrm{~A} / \mathrm{F}$ & Trajectory info \\
\hline \hline 1 & 1 & 5 & 3 & 4.56 & $3 / 0$ & $40 \mathrm{~m}$, up \\
\hline 2 & 4 & 13 & 7 & 3.57 & $4 / 1$ & $70 \mathrm{~m}$, down \\
\hline 3 & 3 & 16 & 5 & 4.89 & $0 / 1$ & $110 \mathrm{~m}$, up \\
\hline 4 & - & - & - & - & - & - \\
\hline
\end{tabular}

\subsection{Formation of Trapezoids}

The sink node begins the process of creating clusters with the support of TFA and AUV. AUV is deployed at fixed depth levels of the ocean and traverses across the linear trajectory. At each level of the linear trajectory, TFA activates the trapezoid formation model to divide the horizontal plane into many vertical slabs whenever the anchor nodes come within the transmission range of AUV, and it stores the anchor nodes' $x$ coordinate in a structured manner on the array, to create SDS. TFA divides each vertical slab further into trapezoids, with the assistance of reference/anchor nodes. Each area of the vertical slab between two consecutive edges forms a unique trapezoid. Some trapezoids border on the seafloor and on the sea surface within the bounding box boundary regions. Vertical lines and the anchor nodes' edges passing through the endpoints create geometrical shapes such as trapezoids, unbounded trapezoids and triangles, as shown in Fig. 6.

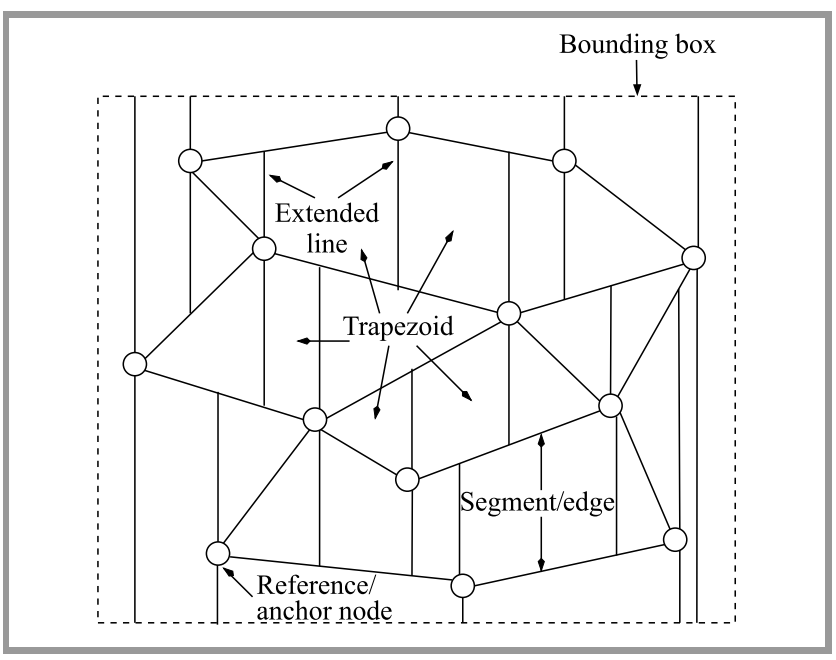

Fig. 6. Trapezoidal map.

A binary search is performed to determine the trapezoid of the uw-sensor node, and $\mathrm{x}$ coordinates are stored in the array, as shown in Fig. 8 [22]. Provisionally, the diagram contains a set of non-crossing line-segments, $\mathrm{S}=$ $\left\{s_{1}, s_{2}, \ldots, s_{n}\right\}$, embedded in the bounding box $\mathrm{R}$ and characterized by the fact that any two anchor nodes have different $\mathrm{x}$ coordinates. If the arrangement comprises a set of non-crossing edges having different anchor nodes' $x$ coordinates and surrounded by fixed limits, then the process of creating a trapezoidal map is more straightforward. TFA creates the trapezoidal map $\mathrm{T}(\mathrm{S})$ using the non-crossing edges of the anchor nodes on the ocean's surface. TFA enlarges two perpendicular edges from each anchor node, one in the ascending direction and the other in the descending direction, until they reach either the edge or the border of $\mathrm{S}$, as shown in Fig. 7 of the trapezoidal map. In a scenario

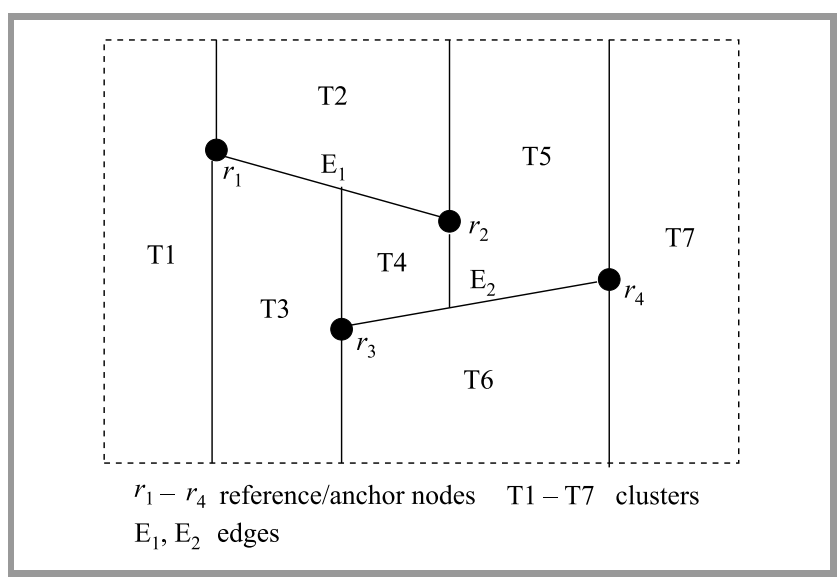

Fig. 7. Two segments trapezoidal map. 
in which any two trapezoids are located next to each other, then such trapezoids share a perpendicular edge. A double connected edge is used in the formation of the trapezoidal map.

During the process of creating the trapezoids, TFA develops SDS $D$, as shown in Fig. 8. Its properties are:

- SDS is a directed-acyclic-graph (DAG),

- SDS is a single-rooted, and each trapezoid is rendered by a leaf node in the trapezoidal-map of $\mathrm{S}$,

- inner nodes' out-degree is two: $x$-node and $y$-node, where $x$-node shows with the endpoints of segments in $S$ and $y$-node represents the segment itself.

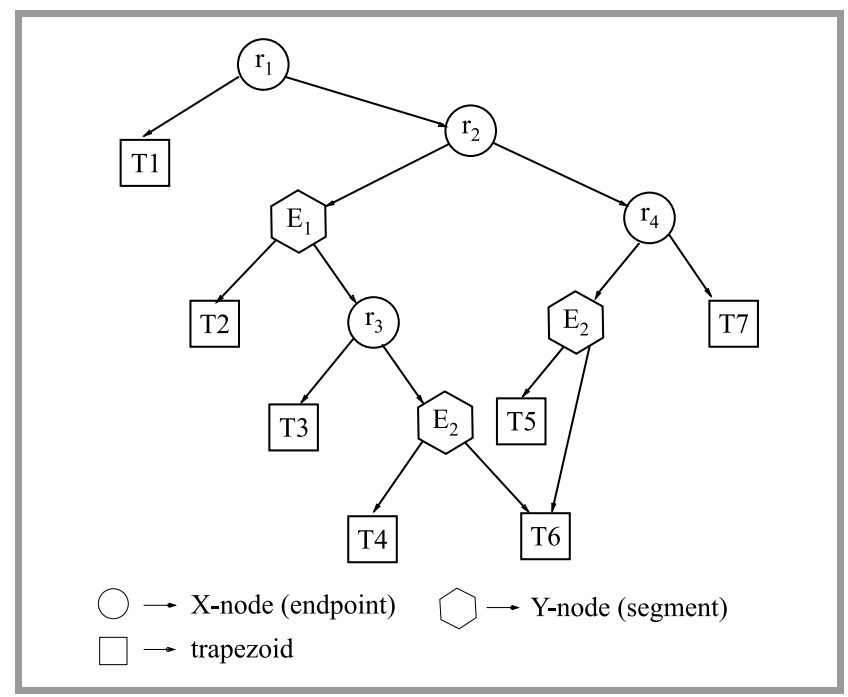

Fig. 8. Search data structure.

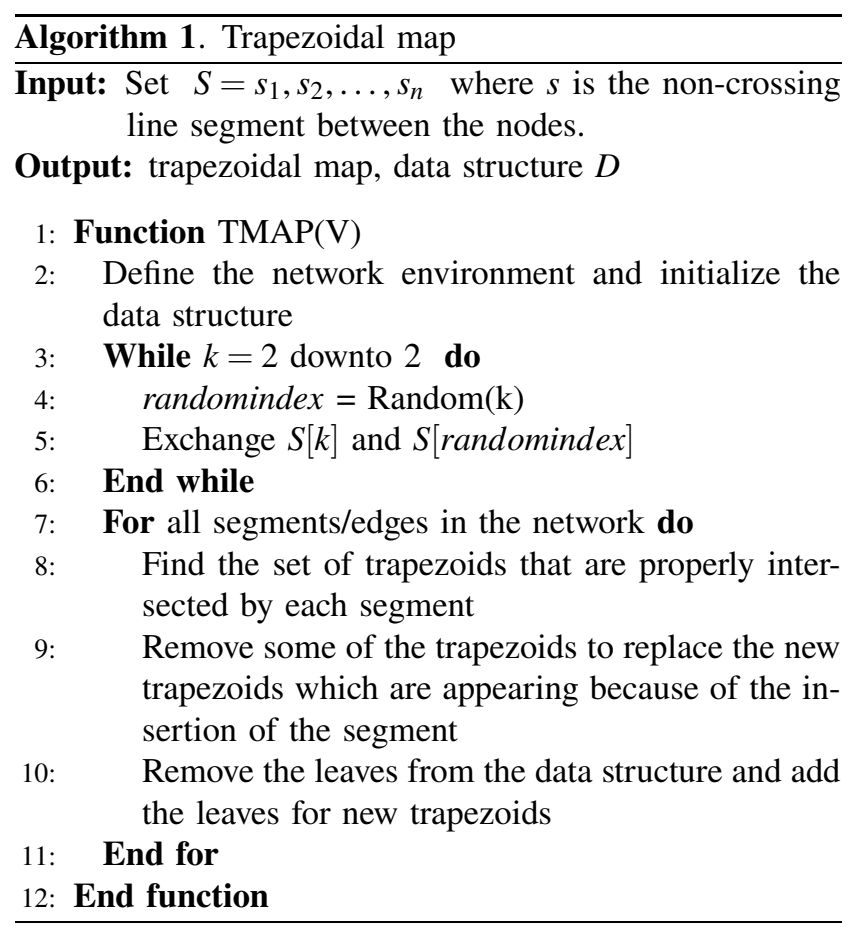

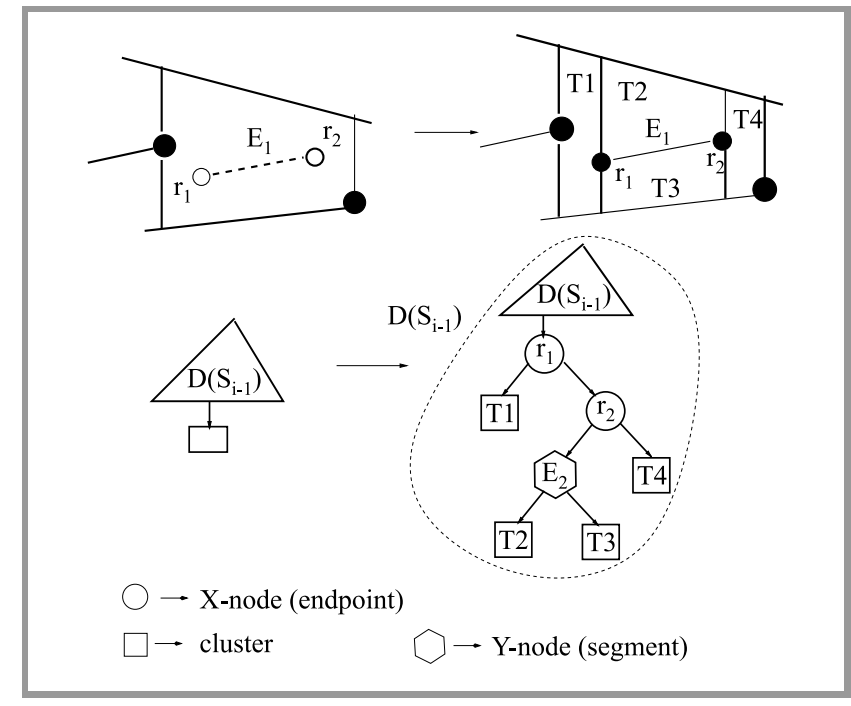

Fig. 9. Data structure used for adding a new segments by replacing trapezoids.

The trapezoidal map and SDS are interlinked with each other. Algorithm 1 describes the process of constructing the trapezoidal map the network. When two anchor/reference nodes, such as $r_{1}, r_{2}$, are positioned entirely in any of the trapezoids, TFA removes some trapezoids and replaces them with new trapezoids, as presented in Algorithm 1, and the related search data structure is constructed as shown in Fig. 9 [22].

\subsection{AUV Trajectory}

The AUV moves along the continuous trajectory at distance $l$ from the initial position $T_{\text {start }}$, until the end position $T_{\text {end }}$. It moves horizontally, at fixed underwater levels, at a constant speed. At each depth, AUV periodically transmits, omnidirectionally, beacon messages including realtime position information. It does so at fixed intervals and with full communication power, as depicted in Fig. 10. The migrant LA activates the localization model at each

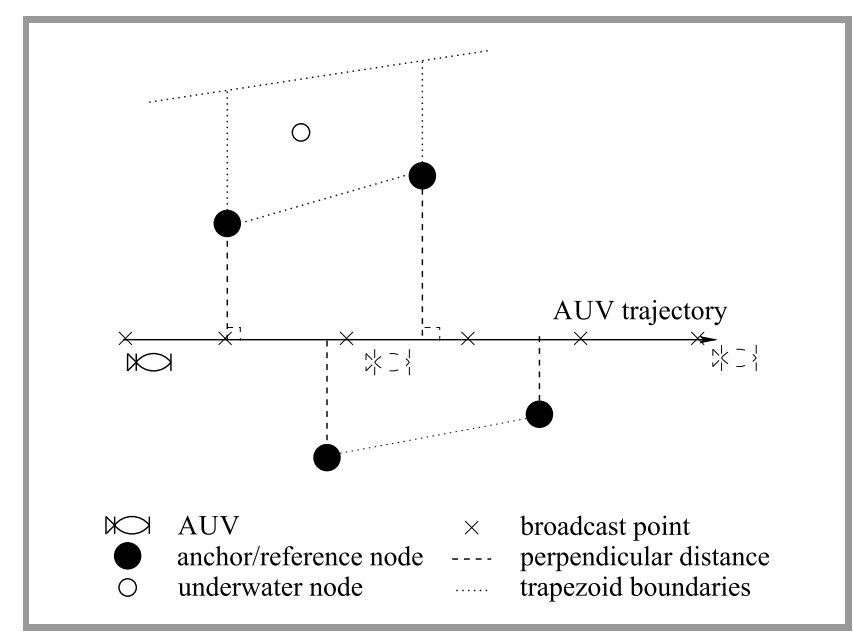

Fig. 10. The trajectory of AUV. 


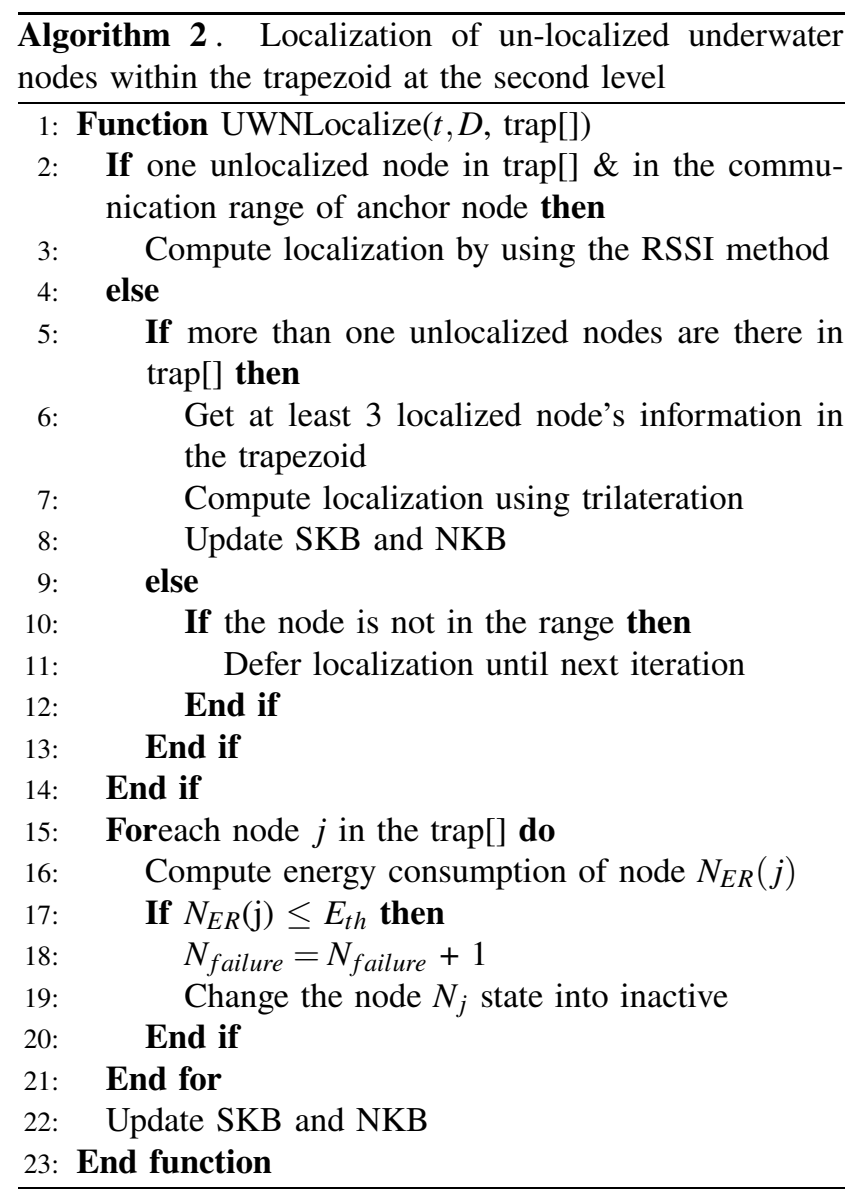

anchor node to receive beacon messages and calculates the received-signal-strength (RSS) of each beacon message. LA estimates the received RSS value in the network environment as:

$$
R_{S L}=S L+N L-P L(l),
$$

where $S L$ is the source level, $N L$ is the noise level, $P L(l)$ is the propagation loss for an acoustic signal in underwater conditions, given as:

$$
\begin{gathered}
N L(f)=N_{t n}(f)+N_{w}(f)+N_{s}(f)+N_{t u}(f)[\mathrm{Pa} / \mathrm{Hz}] \\
S L=10 \log \frac{I_{t}}{1 \mu P a}
\end{gathered}
$$

where $I_{t}$ is the the intensity of the signal transmitted from the broadcast point, at a distance of $1 \mathrm{~m}$ measured in $\mu \mathrm{Pa}$. The $I_{t}$ yield as per [24] is:

$$
I_{t}=10^{\frac{S L}{10}} \cdot 0.67 \cdot 10^{-68}
$$

in watts $/ \mathrm{m}^{2}$. The propagation loss is:

$$
10 \log P L(l, f)=k \cdot 10 \log (l)+l \cdot \alpha(f)+A,
$$

where $k$ is the geometrical spreading constituent of the distribution, $\alpha(\mathrm{f})$ is the absorption pattern shown in Eq. (19), and $A$ is the communication irregularity that eliminates con-

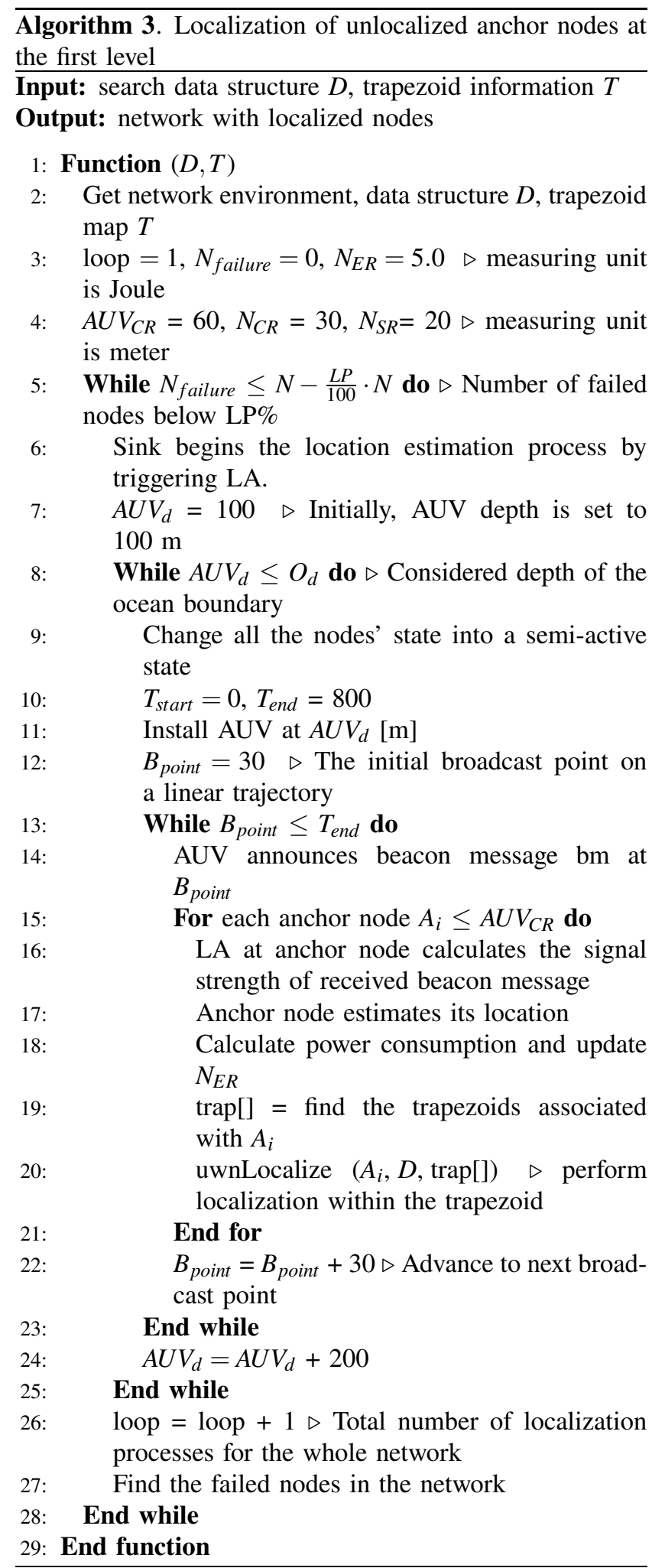

sumption factors and involves the refraction, diffraction, and scattering.

In general, the spreading factor of $k=1.5$ is assumed in this work. Spreading loss is wavelength-independent, and it has the rounded or cylindrical spreading.

Thorp's formula [25] describes $\alpha(\mathrm{f})$ - frequency absorption model as: 


$$
\begin{aligned}
\alpha(f)=\left(0.11 \frac{f^{2}}{f^{2}+1}\right. & +44 \frac{f^{2}}{f^{2}+4100} \\
& \left.+2.75 \cdot 10^{-4} f^{2}+0.003\right) \cdot 10^{-3}
\end{aligned}
$$

Consider any announcement position is $(x, y, z)$ along the AUV's linear path at each depth level, and $\left(x_{r}, y_{r}, z_{r}\right)$ being any anchor node position on the ocean's surface. $z$ is 0 , and the $y$ coordinate is linearly expressed by $x$ due to the AUV's path, which is linear on the ocean's surface. LA gets the RSSI value vector of all announcement points and the $x$ coordinate vector of the announcement points and estimates the position of an anchor node by using RSSI. Once the reference/anchor nodes have learned their locations, LA visits each trapezoid and calculates the location of unlocalized nodes, as described in Algorithm 2. Algorithm 3 describes the process of estimating the location of nodes.

\subsection{Single-node Localization}

If a node moves within the trapezoid due to underwater currents or other aquatic characteristics, then NA may demand localization by asking the anchor node. The anchor node initiates the location estimation process by triggering LA and the procedure is given in Algorithm 4.

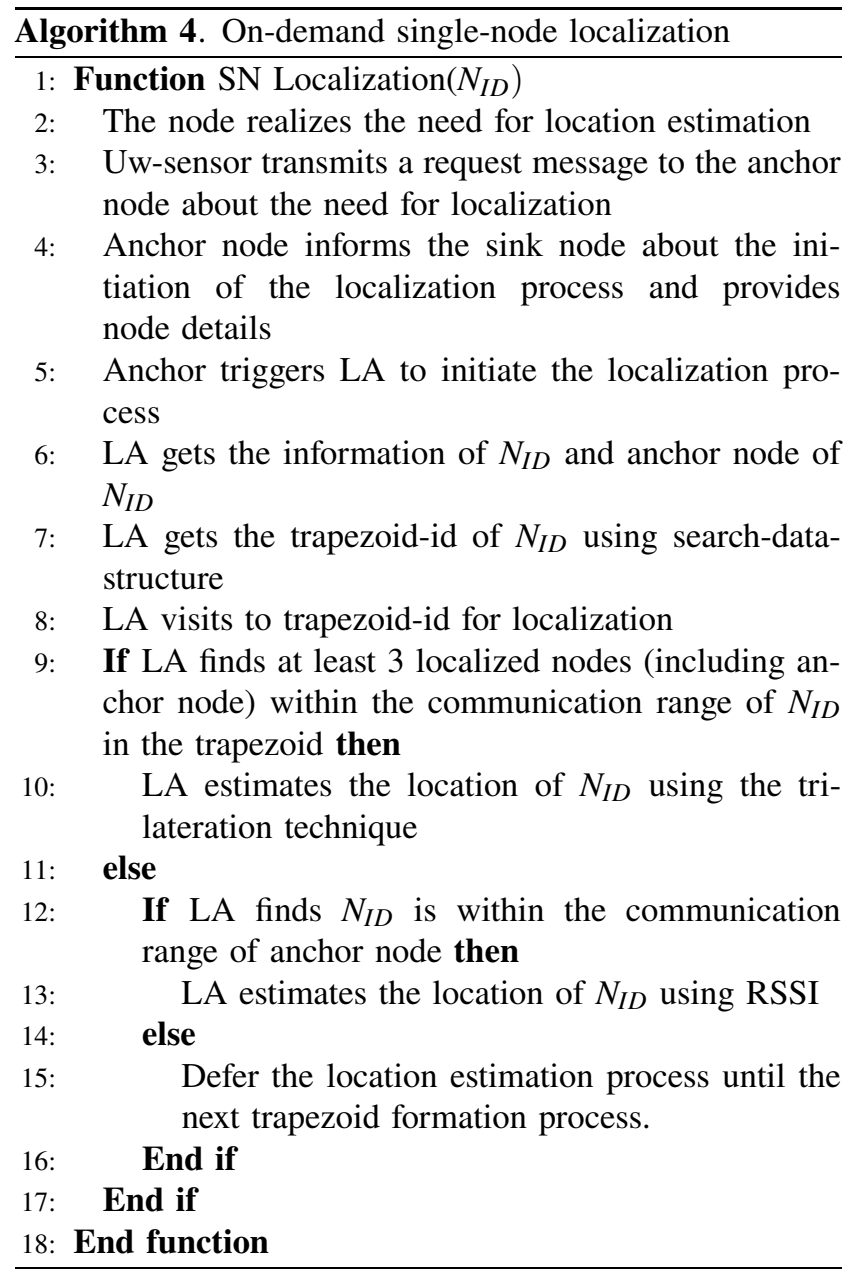

The sink node initiates all-node localization at regular intervals. The scheme is as below:

1. The sink node begins the trapezoid formation activity at each iteration by TFA. TFA divides the region into perpendicular slabs and further divides these into trapezoids. TFA counts the number of localized and unlocalized nodes present in each trapezoid. TFA stores relevant information in SKB, i.e. information storage of the sink node, and also creates search data structure $D$.

2. The sink node begins the localization process through LA and deploys AUV at various depth levels. AUV traverses along the linear trajectory from starting point $T_{\text {start }}$ to endpoint $T_{\text {end }}$ at each depth, with a constant speed. AUV transmits beacon messages including real time location information at fixed distances and with a fixed transmission power, as depicted in Fig. 10. At the anchor node, the LA receives these messages and executes the localization model to estimate its location.

3. The anchor agent at each anchor node initiates the localization process in its assigned trapezoids based on the data given by TFA and SDS $D$.

4. At each trapezoid, LA performs the localization process in the following manner:

- if the node is already localized, then LA updates the localization knowledge base with such information as uw-sensor node ID, uw-sensor node energy level, trapezoid ID, adjacent trapezoids, etc. LA changes its state from semi-active to active;

- if the node is unlocalized, then LA looks for at least three localized nodes which are there in the trapezoids, within the communication range, completes the localization process by using the trilateration technique and changes the node status into active;

- if the uw-sensor node is unlocalized and it is within the communication range of the anchor node, then LA applies the RSSI method to estimate its location;

- if an unlocalized uw-sensor node exists in the trapezoid and it is out of the communication range of the anchor node and of other nodes, then the localization process is deferred until the next iteration;

- the above steps are repeated to localize other nodes in the trapezoid. LA changes the state of all localized nodes from semi-active to active.

5. LA updates location-related information in SKB, $\mathrm{AKB}$, and NKB for future use. 
6. Steps 1-5 are repeated until AUV reaches $T_{\text {end }}$ of each linear trajectory.

7. AUV is deployed at the next vertical level and repeats steps $1-6$ to cover the entire targeted area.

8. At the end of each localization period, all the underwater sensor nodes change their status to semi-active in order to preserve their energy levels.

Algorithm 2 describes the localization process within the trapezoids.

\subsection{Mobility in the Localization Problem}

Movement of the nodes is unavoidable due to underwater currents and other underwater conditions. Each NA maintains $\mathrm{NKB}$, containing such information such as energy level, neighbor count and location points. The movement of a node is severe in shallow waters due to numerous human activities and unpredictable behavior of the sea environment. On the contrary, in deep water, most of the time, the movement of the nodes is not present at all or is very much restricted. The agent may perform self-localization in deep water by itself, provided the node is in communication with at least one node which knows its location. If node A is already localized with $N_{A_{(x, y)}}$ points and if NA observes that the node has changed its position, then LA at the node performs re-localization, with the process explained below:

1. LA obtains the location of the previous uw-sensor node from the NKB and stores it as $N P_{A_{(x, y)}}$, and $N P_{A_{\text {depth }}}$ (i.e. NP is the previous location of the node).

2. LA computes the distance to the uw-sensor node, i.e. $N_{\text {dist }}=N_{\text {speed }} \cdot N_{\delta t}$, where $N_{\delta t}$ is the time difference between the last localized time and the agent's observation time, $N_{\text {speed }}$ is the speed of the uw-sensor node, $N_{\text {dist }}$ is the distance which is scalable. The current position of the node is calculated as:

- assign $N C_{A_{\text {depth }}}=\operatorname{Get}\left(N_{D}\right), \mathrm{NC}$ is the current location of the uw-sensor node,

- compute $d=N P_{A_{d e p t h}}-N C_{A_{d e p t h}}$,

- test if $(d \leq 0)$ and then $N C_{A_{y}}=N P_{A_{y}}+d$, i.e. the node is moved downwards down,

- test if $(d \geq 0)$ and then $N C_{A_{y}}=N P_{A_{y}}-d$, i.e. the node moved upwards.

3. Now, the location of mobile node $\mathrm{A}$ is $\left(N C_{A_{\text {? }}}, N C_{A_{y}}\right)$, where the $x$ coordinate is unknown. The distance formula may be applied to find out the $x$ coordinate if the mobile node is capable of communicating with at least one localized uw-sensor node, i.e. node B with location $\left(N C_{B_{x}}, N C_{B_{y}}\right)$.

4. Find distance $D$ between nodes A and B using RSSI.

5. RSSI $=$ antenna gain + transmit power - path loss.
6. For non-mobile nodes $\mathrm{A}$ and $\mathrm{B}$, antenna gain and transmit power are both constant. Path loss is the function of distance $d$. Hence, RSSI $=f(d), d=$ $f^{\prime}(\mathrm{RSSI})$.

7. For simplicity, consider node A points as $\left(x_{1}, y_{1}\right)$ instead of $\left(N C_{A_{?}}, N C_{A_{y}}\right)$ and node B points as $\left(x_{2}, y_{2}\right)$ instead of $\left(N C_{B_{x}}, N C_{B_{y}}\right)$ Calculate the unknown $x_{1}$ value by:

$$
d=\sqrt{\left(x_{2}-x_{1}\right)^{2}+\left(y_{2}-y_{1}\right)^{2}} .
$$

By taking a square on both the sides, we get:

$$
d^{2}=\left(x_{2}-x_{1}\right)^{2}+\left(y_{2}-y_{1}\right)^{2},
$$

and:

$$
d^{2}-\left(y_{2}-y_{1}\right)^{2}=\left(x_{2}-x_{1}\right)^{2},
$$

where $d, y_{1}, y_{2}$ are known values. After solving RHS, a constant value $v$ becomes:

$$
v=\left(x_{2}-x_{1}\right)^{2} \text {. }
$$

After removing the square on both sides Eq. (23) becomes:

$$
\sqrt{v}=\left(x_{2}-x_{1}\right)
$$

then:

$$
x_{1}=x_{2}-\sqrt{v} .
$$

Now, the $x$ coordinate of node $\mathrm{A}$ is computed and the $y$ coordinate is computed in Step 2 .

8. The agent performed internal localization, and the new coordinates of mobile node $\mathrm{A}$ are $\left(N C_{A_{x}}, N C_{A_{y}}\right)$.

The proposed work highlights the use of computational geometry for estimating the location of unlocalized nodes. It is supported by agent technology, which supports various APIs for during the implementation phase. Modules for trapezoid creation, SDS establishment and trajectory path formation are designed using mathematical models and may be implemented with suitable modifications. Anchor/reference nodes are considered at each stage of the proposed work, and are therefore included in trapezoid creation, trajectory path formation, SDS creation and location estimation modules. Regular nodes predominantly included in location estimation and mobility modules.

\section{Simulation}

The proposed uw-sensor node localization method is simulated and assessed based on various parameters. At the initial stage of the simulation, all nodes are considered to be characterized by equal power, equal sensing range and equal transmission capability. The sink node switches all nodes into semi-active state to preserve energy. The node location estimation process is performed iteratively. At each step, 
most of the nodes attempt to localize themselves and consume energy. The simulation is terminated when the energy level of $70 \%$ of all nodes falls below a specific threshold value. Simulation models, procedures and performance parameters are discussed in this section.

\subsection{Simulation Model}

A monitored area with the size of $600 \times 600 \times 600 \mathrm{~m}$ is used for UASN simulation purposes. Initially, uw-sensor nodes are deployed randomly, beginning with 20 and with their number increasing to 100 within the $3 \mathrm{D}$ space. Initially, the sink node deploys AUV at $\mathrm{AUV}_{d}$ and it then moves along a continuing trajectory. AUV broadcasts location information at regular intervals, $5 \mathrm{~L}$ is set to $30 \mathrm{~m}$. At each vertical level, AUV travels linearly over a distance of $600 \mathrm{~m}$, with fixed frequency $T_{f}=24 \mathrm{kHz}$, sound level $\mathrm{SL}=100 \mathrm{~dB}$ and spreading factor $k=1.5$. A few anchor nodes are deployed randomly. A beacon message advances linearly at a velocity of one $\mathrm{m} / \mathrm{s}$ and makes announcements one second intervals. The sink node is installed onshore. In the propagation model, sensing range $N_{S R}$, communication range $N_{C R}$ of an acoustic UASN node for single hop communication and attenuation factor $\alpha(f)$ are given in Eq. (19), as per Ainslie and McColm [26].

Table 5

Simulation input data

\begin{tabular}{|l|c|}
\hline \multicolumn{1}{|c|}{ Parameter } & Value \\
\hline \hline Width $w$ & $600 \mathrm{~m}$ \\
Length $l$ & $600 \mathrm{~m}$ \\
Depth $d$ & $600 \mathrm{~m}$ \\
Uw-sensor nodes $n$ & 100 \\
Uw-sensor node communication range $N_{C R}$ & $30 \mathrm{~m}$ \\
Temperature range & $2-20^{\circ} \mathrm{C}$ \\
AUV communication range $A U V_{C R}$ & $60 \mathrm{~m}$ \\
Anchor nodes & 30 \\
Transmission frequency $T_{f}$ & $24 \mathrm{kHz}$ \\
Attenuation $\alpha$ & $0.01-1.0$ \\
SL & $100 \mathrm{~dB}$ \\
$\delta \mathrm{L}$ & $30 \mathrm{~m}$ \\
Speed of ship $v$ & $5 \mathrm{~m} / \mathrm{s}$ \\
$k$ & 1.5 \\
\hline
\end{tabular}

The performance parameters are:

Localization ratio. It is the ratio of localized uw-sensor nodes to the total number uw-sensor nodes in the network.

Energy consumption. It is calculated as total power consumption of all nodes in one iteration. To obtain intensity $I_{t}$ with communication power $P_{t}$ at an interval of $1 \mathrm{~m}$ from the origin towards the recipient as per [24], the following equation is used:

$$
P_{t}=2 \pi D / t \cdot 1 \quad[\mathrm{~m}],
$$

in watts, where $D$ is the depth measured in meters and $I_{t}$ is taken from Eq. (17).

In each trapezoid, power consumption of $m$ localized uwsensor nodes involved in the trilateration of packet size $P_{\text {size }}$, is:

$$
P_{c}=\frac{m P_{\text {size }}}{m 1024}=\frac{P_{\text {size }}}{1024}
$$

in watts/bit. Assume $T_{\text {tactive }}$ is the total active time of a uwsensor node's transceiver in seconds, per one iteration, $N_{E R}$ is the residual energy available at every uw-sensor node in $\mathrm{w} / \mathrm{h}$. The ratio of the total residual energy to the power required for one packet is the total active time of the transceiver and is given by:

$$
T_{\text {active }}=\frac{N_{E R}}{P_{c}} .
$$

Since the uw-sensor node operates in different states, its transceiver's active period equals A seconds, and the node's battery life is given by:

$$
T_{\text {lifetime }}=\frac{T_{\text {active }}}{T_{\text {tactive }}} \cdot \frac{A}{24 \cdot 60} \quad[\text { days }] .
$$

Localization accuracy. It's the difference between the original and the estimated location. If $N E_{(x, y, z)}$ is the calculated location of node and $N A_{(x, y, z)}$ is the original location, localization accuracy is given as:

$$
L E=\left|N E_{(x, y, z 0}-N A_{(x, y, z)}\right| \text {. }
$$

The average location error is:

$$
L E_{\text {avg }}=\frac{1}{n} \sum_{i=1}^{n} L E_{i} .
$$

The location error is a vital factor if it is larger than a specified threshold value.

Network lifetime. It is the number of times the location estimation process is performed until the energy level of $70 \%$ of the deployed uw-sensor nodes falls below a specified threshold value. To calculate it, we need to first obtain the number of uw-sensor nodes $m$ whose power level is greater than the threshold value, i.e. the number of nodes $m$ if $\left(T_{\text {lifetime }}(i) \leq E_{t h}\right)$ for every node $i$ in the system. To ensure the network is connected, let $P_{N}$ be the percentage share of uw-sensor nodes whose energy level is higher than the threshold value. Here, $T_{\text {lifetime }}$ is the total lifetime of a uw-sensor node:

$$
N_{\text {stable }}=\frac{P_{N}}{100} \cdot n
$$

if $m \geq\left(n-N_{\text {stable }}\right)$, the network fails. The location estimation process is repeated after a specific condition is satisfied.

\subsection{Simulation Procedure}

The proposed MASD scheme relies on specific simulation parameters and the simulation process is repeated until $70 \%$ 
of the uw-sensor nodes retain energy levels that are higher than a specific threshold value. Algorithm 5 presents the simulation procedure using pseudo-code.

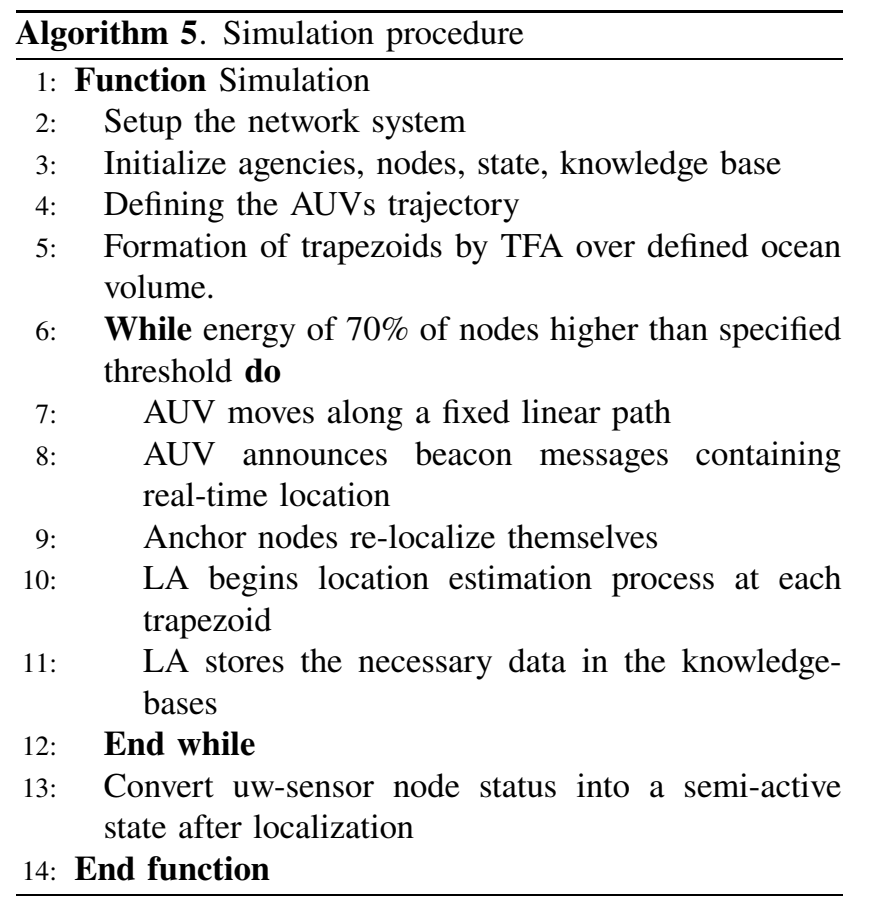

\section{Analysis of Results}

In the proposed scheme, the algorithm is focused on creating trapezoids with the help of an internal search data structure. Figures 11 and 12 show the number of trapezoids created with a fixed number of 40 and 80 uw-sensor nodes, respectively. A higher number of trapezoids is observed in shallow waters, due to the denser deployment of uw-sensor nodes. In a scenario with a lower number of reference nodes, the formation of trapezoids is restricted due to greater gaps between the positions of reference nodes. If the number of reference nodes is increased, the number of trapezoids of various sizes present in the network increases as well. In the case shown in Fig. 12, it is observed that a higher number of trapezoids is created because

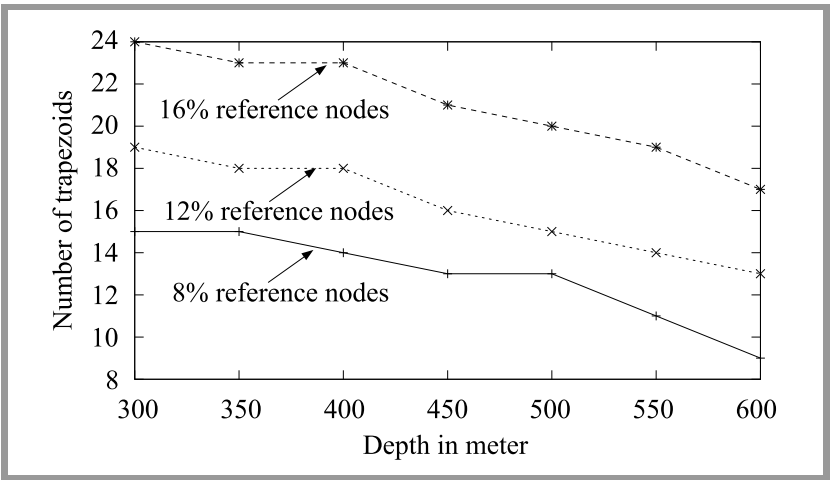

Fig. 11. Number of trapezoids created for 40 nodes at various depths.

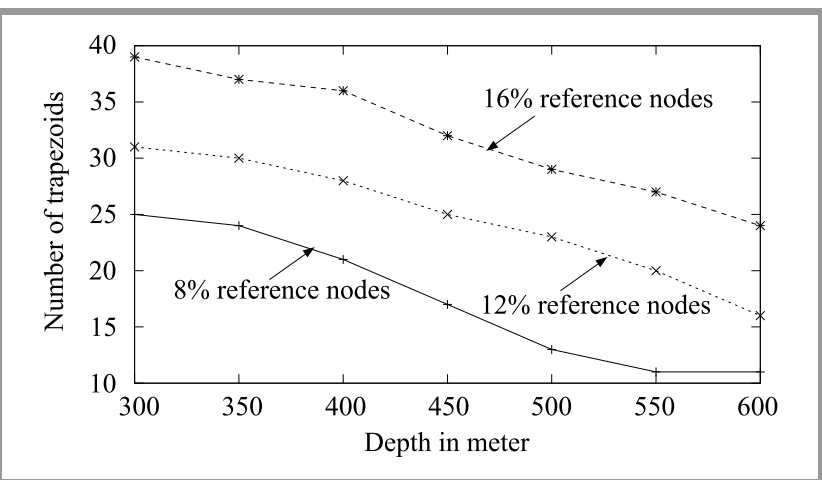

Fig. 12. Number of trapezoids created for 80 nodes at various depths.

of the deployment of more uw-sensor nodes. If more trapezoids are created, the lifetime of the network is affected, but it becomes easier to localize uw-sensor nodes. The sink node also participates in the creation of SDS, as shown in Fig. 8.

\subsection{Localization Accuracy}

Figure 13 shows that the location error is higher in the case of the LDB scheme than in the proposed scheme. In the recommended scheme, agents broadcast beacon messages

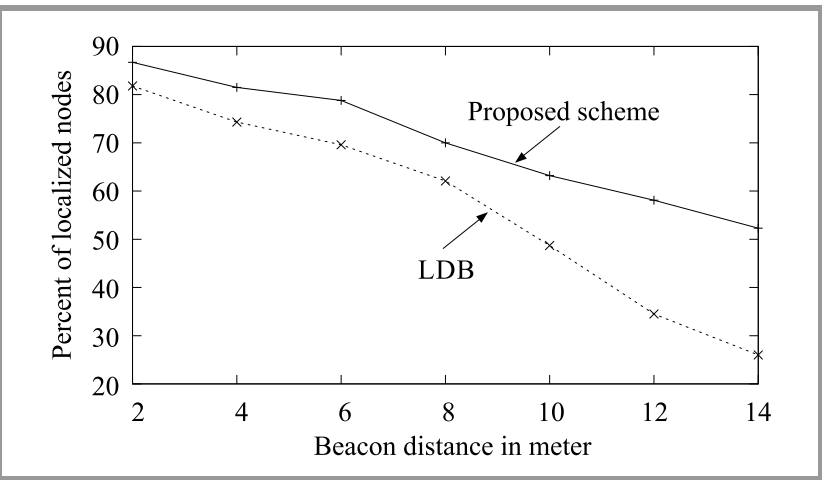

Fig. 13. Localization accuracy.

at regular intervals of variable beacon distance. In the proposed scheme, the size of the trapezoid is measurable and is well defined by anchor nodes, making it easier to localize the unlocalized nodes with a minimum error rate. The SDS identifies the node's nearest trapezoid to which it belongs. This reduces the error in the actual location and the calculated location. If the beacon distance keeps increasing, the error in the computed position increases as well, as observed in the proposed scheme. Acoustic waves propagate spherically, making them vulnerable to attenuation. Hence, the position of sensor nodes is estimated with a minor error rate. Location estimation depends also on the nodes' movement in the undersea environment, caused by water currents. In this calculation, the mobility factor is not taken into consideration. 


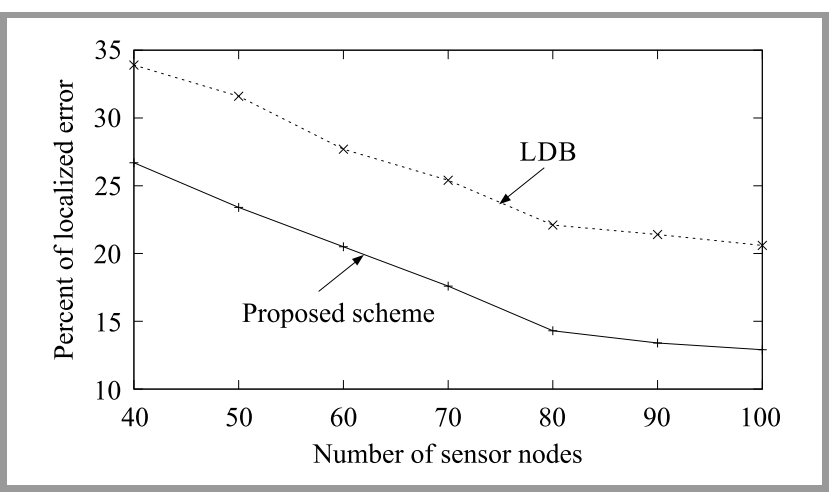

Fig. 14. Localization error.

Figure 14 shows the impact of the change in the number of uw-sensor nodes on the localization error. The proposed scheme renders better results in terms of minimizing the localization error, even if the number of nodes increases. It results in a higher level of availability of nodes in each trapezoid, which facilitates trilateration. It is observed that the sufficient availability of nodes in each trapezoid reduces the localization error. Such an approach consumes more power due to excessive communication between the nodes. It is observed that the location error does not varies much in the event of an increase in the number of uw-sensor nodes beyond a specific limit value.

\subsection{Network Lifetime}

Figure 15 shows network lifetime as a function of active nodes in the network. Network lifetime for the proposed MASD scheme is better than in the LDB method. In a scenario with a higher number of reference nodes, network lifetime is shorter due to the fact that the nodes are involved, on numerous occasions, in the location estimation process. Once the nodes have been localized, then their status needs to be changed to semi-active in order to improve network endurance. In the case of fewer reference nodes, network lifetime is comparatively better than in the case of other schemes. Nodes positioned in shallow water are more mobile due to the presence of water currents and require frequent localization, which reduces network lifetime.

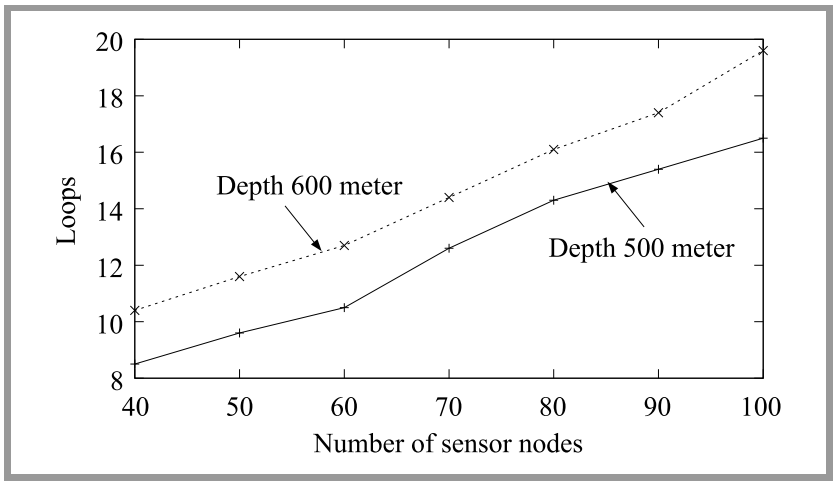

Fig. 15. Network lifetime.

\subsection{Localization Ratio}

As depicted in Fig. 16, the localization ratio depends, to a higher degree, on the deployment of reference nodes within the undersea network. The location estimation ratio improves linearly with an increase in the number of reference nodes. The proposed MASD scheme proves that more non-localized uw-sensor nodes are then localized in large numbers and, hence, the localization ratio is better than in the LDB scheme. In the proposed method, many trapezoids are formed and each reference node has a common vertex with several trapezoids. Therefore, each reference node is participating in the location estimation process with its dependent trapezoids. In larger structure trapezoids, it is not easy to localize unlocalized nodes because of the transmission range of reference nodes, which causes a reduction in the localization ratio. However, trapezoidal map and the search data structure allow the proposed scheme to achieve a better localization ratio.

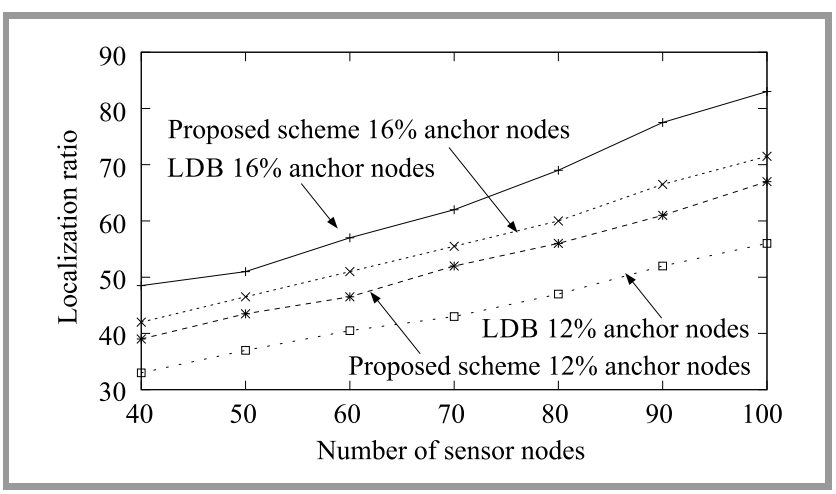

Fig. 16. Localization ratio.

\subsection{Energy Consumption}

Energy consumption of the presented scheme is lower, in the case of a small number of reference nodes, than in other schemes (Fig. 17). The proposed MASD scheme depletes more power (compared to the LDB scheme) in a scenario with more reference nodes because of the unnecessary participation of nodes in the location estimation process. Sensor nodes near the ocean's surface are mobile and require frequent localization, which causes an increase

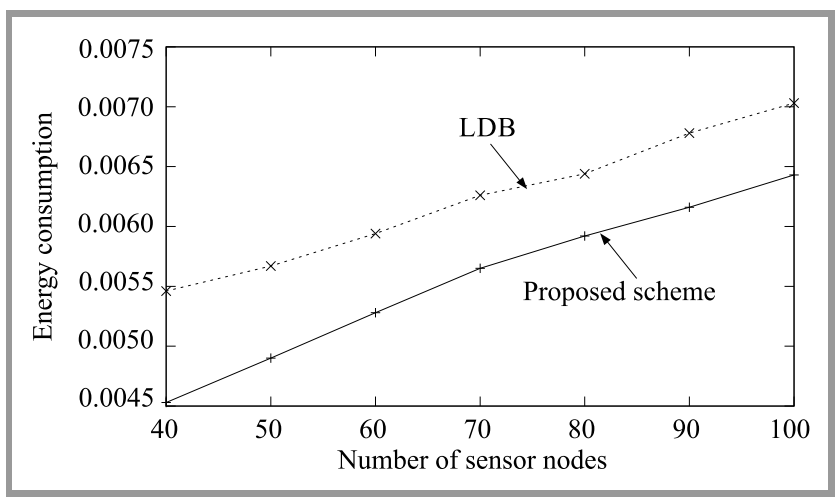

Fig. 17. Localization energy. 
in power consumption. At higher depth levels, mobility of uw-sensor nodes is restricted and, hence, frequent localization of uw-sensor nodes is not required. The graph below shows energy consumption with several nodes.

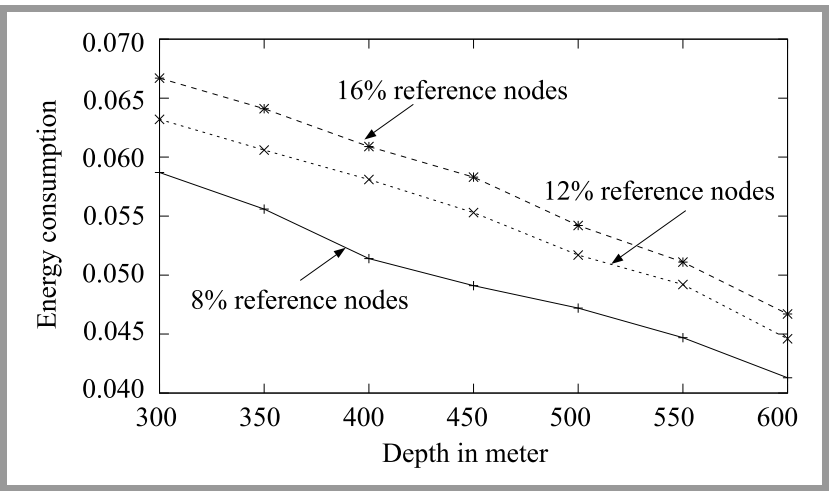

Fig. 18. Energy consumed by nodes in each trapezoid for 40 nodes.

Figure 18 presents the amount of energy consumed by uwsensor nodes in each trapezoid, at various depth levels, for a fixed number of $40 \mathrm{uw}$-sensor nodes. At the depth of $300 \mathrm{~m}$, the reference nodes form several trapezoids of variable sizes. This means that more nodes are present in each of the trapezoids and that many nodes are involved in the localization process. The recursive involvement of nodes in the localization process leads to higher power consumption. If the depth is increased further, the number of trapezoids formed decreases and fewer uw-nodes are present in such trapezoids. Redundant involvement of the nodes in the localization process is minimized and energy consumption of the nodes decreases, as shown in the graph.

Figure 19 shows the energy dissipated by uw-sensor nodes in each trapezoid, with changing water depth and a fixed number of 80 nodes. In shallow water, reference nodes form some trapezoids of variable sizes. The scenario evolves into one characterized by higher density of nodes in each of the trapezoids, with a higher number of nodes involved in the localization process, compared to Fig. 18. The recursive engagement of nodes in the localization process drives power dissipation, which is higher than that

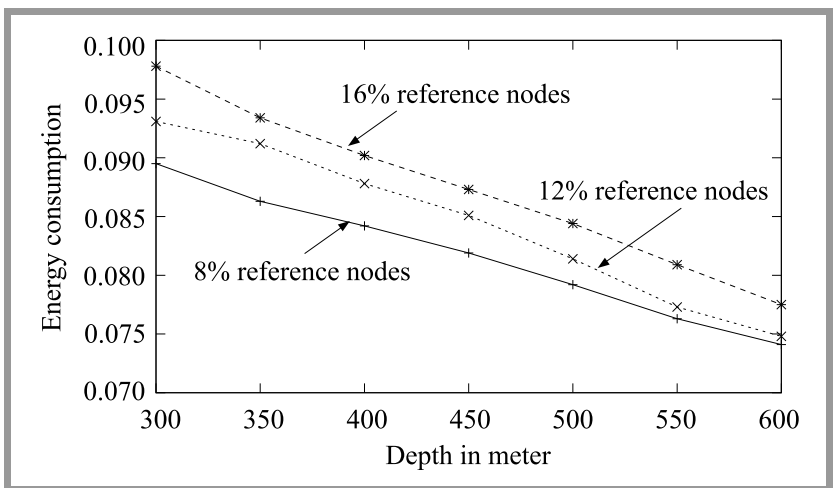

Fig. 19. Energy consumed by nodes in each trapezoid (for 80 nodes). shown in Fig. 18. If the depth is increased further, fewer trapezoids are formed and fewer uw-nodes are present in such trapezoids. The nodes' excessive involvement in the localization process is moderately reduced and minimizes energy consumption, as observed in the graph.

\section{Conclusion}

In this paper, a node location estimation method is presented relying on computational geometrical trapezoids. The proposed scheme uses the trapezoids to create clusters of various sizes. The entire network is divided into trapezoids and a search data structure (SDS) is established. Further location estimation techniques are applied to localize nodes. Simulations performed prove that the proposed scheme may be used in UASN networks, offering good performance and accuracy levels.

\section{References}

[1] Z. Zhou, Z. Peng, J. Cui, and A. Bagtzoglou, "Scalable localization with mobility prediction for underwater sensor networks", IEEE Trans. on Mob. Comput., vol. 10, no. 3, pp. 335-348, 2011 (DOI: 10.1109/TMC.2010.158).

[2] X. Cheng et al., "Silent positioning in underwater acoustic sensor networks", IEEE Trans. Veh. Technol., vol. 57, no. 3, pp. 1756-1766, 2008 (DOI: 10.1109/TVT.2007.912142).

[3] Y. S. Uddin, "Low-overhead range-based 3D localization technique for underwater sensor techniques", in Proc. of IEEE Int. Conf. on Commun. ICC 2016, Kuala Lumpur, Malaysia, 2016 (DOI: 10.1109/ICC.2016.7510873).

[4] X. Sheng and Y.-H. Hu, "Maximum likelihood multiple-source localization using acoustic energy measurements with wireless sensor networks", IEEE Trans. on Sig. Process., vol. 53, no. 1, pp. 44-53, 2005 (DOI: 10.1109/TSP.2004.838930).

[5] V. Chandrasekhar and W. Seah, "An area localization scheme for underwater sensor networks", Proc. OCEANS 2006 - Asia Pacific, Singapore, 2006 (DOI: 10.1109/OCEANSAP.2006.4393969).

[6] S. Kundu and P. Sadhukhan, "Design and implementation of a time synchronization-free distributed localization scheme for underwater acoustic sensor network", in Proc. of Appl. and Innov. in Mob. Comput AIMoC 2015, Kolkata, India, 2015, pp. 74-80 (DOI: 10.1109/AIMOC.2015.7083833).

[7] E. E. Lloyd et al., "UREAL: underwater reflection-enabled acousticbased localization", IEEE Sensors J., vol. 14, no. 11, pp. 3915-3925, 2014 (DOI: 10.1109/JSEN.2014.2357331).

[8] R. Hamid et al., "Collision tolerant and collision free packet scheduling for underwater acoustic localization", IEEE Trans. on Wirel. Commun., vol. 14, no. 5, pp. 2584-2595, 2015 (DOI: 10.1109/TWC.2015.2389220).

[9] G. Han et al., "Impacts of deployment strategies on localization performance in underwater acoustic sensor networks", IEEE Trans. on Industr. Electron., vol. 62, no. 3, pp. 1725-1733, 2015 (DOI: 10.1109/TIE.2014.2362731).

[10] T. V. Nguyen et al., "Least square cooperative localization", IEEE Trans. on Veh. Technol., vol. 64, no. 4, pp. 1318-1330, 2015 (DOI: 10.1109/TVT.2015.2398874).

[11] T. Xu et al., "RSS-based sensor localization in underwater acoustic sensor networks", in Proc. of 41st IEEE Int. Conf. on Acoust., Speech and Sig. Process. ICASSP 2016, Shanghai, China, 2016, pp. 3906-3910 (DOI: 10.1109/ICASSP.2016.7472409).

[12] A. Savvides, H. Park, and M. B. Srivastava, "The bits and flops of the N-hop multilateration primitive for node localization problems", in Proc. of the 1st ACM Int. Worksh. on Wirel. Sensor Netw. and Appl. WSNA'02, Atlanta, GA, USA, 2002, pp. 112-121 (DOI: 10.1145/570753.570755). 
[13] A. Savvides, C. C. Han and M. B. Srivastava, "Dynamic fine-grained localization in ad hoc networks of sensors", in Proc. of the 7th Ann. Int. Conf. on Mob. Comput. and Network. MobiCom'01, Rome, Italy, 2001, pp. 166-179, 2001 (DOI: https://doi.org/10.1145/381677.381693).

[14] G. Zhu et al., "A distributed localization scheme based on mobility prediction for underwater wireless sensor networks", in Proc. of the 26th Chinese Contr. and Decision Conf. CCDC 2014, Changsha, China, 2014, pp. 4863-4867 (DOI: 10.1109/CCDC.2014.6853044).

[15] S. Lee and K. Kim, "Localization with a mobile beacon in underwater acoustic sensor networks", Sensors, vol. 12, no. 5, pp. 54865501, 2012 (DOI: 10.3390/s120505486).

[16] Y. Sun et al., "A mobile anchor node assisted RSSI localization scheme in underwater wireless sensor networks", Sensors, vol. 19, no. 20, 2019 (DOI: 10.3390/s19204369).

[17] C. Zhang et al., "A collaborative localization algorithm for UASNs", in Proc. of the Int. Conf. on Comput., Manag. and Telecommun. ComManTel 2014, Da Nang, Vietnam, 2014, pp. 211-216 (DOI: 10.1109/ComManTel.2014.6825606).

[18] J. Gao et al., "A double rate localization algorithm with one anchor for multi-hop underwater acoustic networks", Sensors, vol. 17, no. 5, pp. 984-1001, 2017 (DOI: 10.3390/s17050984.

[19] M. Beniwal, R. P. Singh, and A. Sangwan, "A localization scheme for underwater sensor networks without time synchronization", Wirel. Pers. Commun., vol. 88, no. 3, 2016 (DOI: $10.1007 / \mathrm{s} 11277-016-3175-2)$.

[20] Y. Zhang, J. Liang, S. Jiang, and W. Chen, "A localization method for underwater wireless sensor networks based on mobility prediction and particle swarm optimization algorithms", Sensors, vol. 16, no. 2, pp. 212, 2016 DOI: https://dx.doi.org/10.3390/s16020212.

[21] Xin Su, I. Ullah, X. Liu, and D. Choi, "A review of underwater localization techniques algorithms and challenges", J. of Sensors, vol. 2020, no. 1, pp. 1-24, 2020 (DOI: 10.1155/2020/6403161).

[22] M. de Berg et al., Computational Geometry, 3 ed. Berlin, Heidelberg: Springer, 1983 (ISBN: 9783540779742).

[23] B. Zhang et al., "Received signal strength-based underwater acoustic localization considering stratification effect", in Proc. of the OCEANS 2016, Shanghai, China, 2016 (DOI: 10.1109/OCEANSAP.2016.7485561).

[24] R. J. Urick, Principles of Underwater Sound, 1 ed. New York: McGraw-Hill, 1983 (ISBN: 9780070660878).

[25] W. H. Thorp, "Analytic description of the low frequency attenuation coefficient", J. of Acoustic. Soc. of America, vol. 42, no. 1, pp. 270, 1967 (DOI: 10.1121/1.1910566).

[26] W. Zhang et al., "Fault-tolerant relay node placement in wireless sensor networks: Problems and algorithms", in Proc. of the 26th IEEE Int. Conf. on Comp. Commun. INFOCOM 2007, Barcelona, Spain, 2007, pp. 1649-1657 (DOI: 10.1109/INFCOM.2007.193).

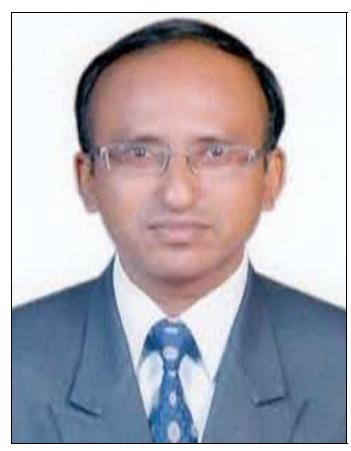

B. S. Halakarnimath received his B.E. and M.Tech. degrees in Computer Science and Engineering from VTU, Belagavi, Karnataka, India. Presently, he is a VTU research scholar pursuing his Ph.D. under the guidance of Dr. A. V. Sutagundar, at Basaveshwar Engineering College, Bagalkot, Karnataka, India. He has authored one book chapter and published five conference and journal papers. His research areas include underwater acoustic wireless sensor networks, machine learning, and algorithms. He is a life member of IEI and ISTE organizations.

E-mail: basaprabhu97@gmail.com

Research Scholar of VTU

Department of Computer Science and Engineering

S.G. Balekundri Institute of Technology

Belagavi-590010, Karnataka, India

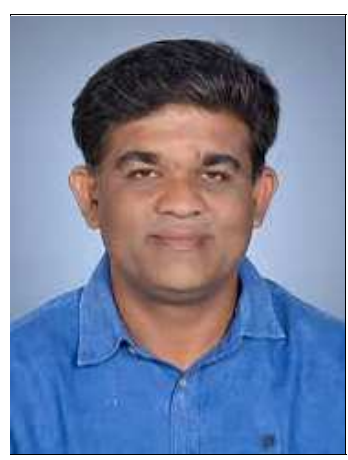

A. V. Sutagundar received his B.E. and M.Tech. degrees in Electronics and Communication Engineering from VTU, Belagavi, Karnataka, India. He received his Ph.D. in 2013 from VTU, Belagavi, India. Presently, he is working as an Associate Professor at the Department of ECE, Basaveshwar Engineering College, Bagalkot, Karnataka, India. He has authored eight book chapters and published more than 75 papers at international conferences and in various journals. His research areas include IoT, wireless sensor networks, machine learning, and digital image processing.

E-mail: sutagundar@gmail.com

Department of Electronics and Communication

Basaveshwar Engineering College

Bagalkot-587102, Karnataka, India 